The Astrophysical Journal, 655:718-734, 2007 February 1

(C) 2007. The American Astronomical Society. All rights reserved. Printed in U.S.A.

\title{
A STRONG CORRELATION BETWEEN CIRCUMNUCLEAR DUST AND BLACK HOLE ACCRETION IN EARLY-TYPE GALAXIES
}

\author{
Ramiro D. Simões Lopes, Thaisa Storchi-Bergmann, and Maria de Fátima Saraiva \\ Instituto de Física, Universidade Federal do Rio Grande do Sul, Porto Alegre, RS, Brazil; ramirosl@if.ufrgs.br \\ AND \\ PAUl Martini \\ Department of Astronomy, The Ohio State University, Columbus, OH; martini@astronomy.ohio-state.edu \\ Received 2006 September 12; accepted 2006 October 9
}

\begin{abstract}
We present a detailed investigation of the incidence of circumnuclear dust structure in a large, well-matched sample of early-type galaxies with and without active galactic nuclei (AGNs). All 34 early-type AGN hosts in our sample have circumnuclear dust, while dust is only observed in $26 \%$ (9) of a pair-matched sample of 34 early-type, inactive galaxies. This result demonstrates a strong correlation between the presence of circumnuclear dust and accretion onto the central, supermassive black hole in elliptical and lenticular galaxies. This correlation is not present at later Hubble types, where a sample of 31 active and 31 inactive galaxies all contain circumnuclear dust. These archival, Hubble Space Telescope observations reveal a wide range of mostly chaotic dust morphologies. Current estimates suggest the dust settling or destruction time is on order of $10^{8} \mathrm{yr}$, and therefore the presence of dust in $\sim 50 \%$ of early-type galaxies requires frequent replenishment and similarly frequent fueling of their central supermassive black holes. The observed dust could be internally produced (via stellar winds) or externally accreted, although there are observational challenges for both of these scenarios. Our analysis also reveals that approximately one-third of the early-type galaxies without circumnuclear dust have nuclear stellar disks. These nuclear stellar disks may provide a preferred kinematic axis to externally accreted material, and this material may in turn form new stars in these disks. The observed incidence of nuclear stellar disks and circumnuclear dust suggests that episodic replenishment of nuclear stellar disks occurs and is approximately concurrent with the fueling of the central AGN.
\end{abstract}

Subject headings: dust, extinction — galaxies: active — galaxies: elliptical and lenticular, $\mathrm{cD}$ galaxies: nuclei - galaxies: structure - ISM: structure

\section{INTRODUCTION}

It is now widely accepted that most, if not all, galaxy bulges host a supermassive black hole (SMBH) at their center (Ferrarese $\&$ Merrit 2000; Gebhardt et al. 2000; Tremaine et al. 2002). However, only a fraction of these galaxies show evidence of significant accretion onto these central black holes in the form of active galactic nuclei (AGNs). The absence of significant accretion in many galaxies may be due to the lack of fuel (most likely in the form of cold interstellar gas), the lack of a fueling mechanism to remove angular momentum and drive this matter toward the center, or both. The most direct probe of the presence of fuel is the direct observation of cold interstellar gas, such as in the form of dust clouds in the central regions of galaxies. Cold dust is also dissipative and therefore sensitive to perturbations that may drive fuel toward the center of the galaxy. Thus, the study of the dust distribution and its dynamical properties may provide valuable information about the powering, transport mechanisms, and timescales of nuclear activity. Identification of the origin of AGN fuel and the nature of the triggering mechanism(s) are two of the main unsolved questions in AGN and black hole research (e.g., Martini 2004).

A large fraction of all galaxies, including those with early-type morphology, have long been known to possess some form of dust structure (e.g., Kormendy \& Djorgovski 1989). The best spatial resolution of the centers of nearby galaxies can be obtained with the Hubble Space Telescope (HST), which reveals the presence of cold interstellar clouds within parsecs of many nearby galactic nuclei. One of the first large HST studies of early-type galaxies by van Dokkum \& Franx (1995) inferred that $78 \%$ of a sample of 64 galaxies contain circumnuclear dust. These authors also noted that the frequency of dust detections is higher in radiodetected galaxies $(72 \%)$ than in galaxies without radio emission (33\%), which suggested a correlation with nuclear activity. More recent $H S T$ studies have also found that dust is common in the centers of early-type galaxies and a correlation between the presence of dust and line-emitting gas and/or AGNs (Ravindranath et al. 2001; Tran et al. 2001; Lauer et al. 2005). For later Hubble types, Malkan et al. (1998) also showed that dust structures were present in most HST WFPC2 images; of a sample of 256 Seyfert and starburst galaxies, only a small fraction of the Seyfert galaxies did not appear to contain dust. Multicolor $V$ - and $H$-band observations of additional Seyferts further showed that circumnuclear dust was always found in the circumnuclear region of the predominantly spiral galaxy hosts of nearby Seyfert 2s (Regan \& Mulchaey 1999; Martini \& Pogge 1999) and Seyfert 1s (Pogge \& Martini 2002).

A more robust way to address the relationship between the presence of circumnuclear gas and/or dust and nuclear activity is to look for differences between AGN host galaxies and a control sample of inactive counterparts. In a recent paper, Xilouris \& Papadakis (2002) searched for dust structures in HST images of a sample of 23 Seyfert and 35 inactive galaxies by fitting and subtracting elliptical isophotes. They found that all active galaxies possess some form of nuclear dust structure and, for their subsample of early-type galaxies, the Seyferts exhibit more nuclear structure than the inactive galaxies, although the opposite seems to be true for the later types. In a more recent paper, 

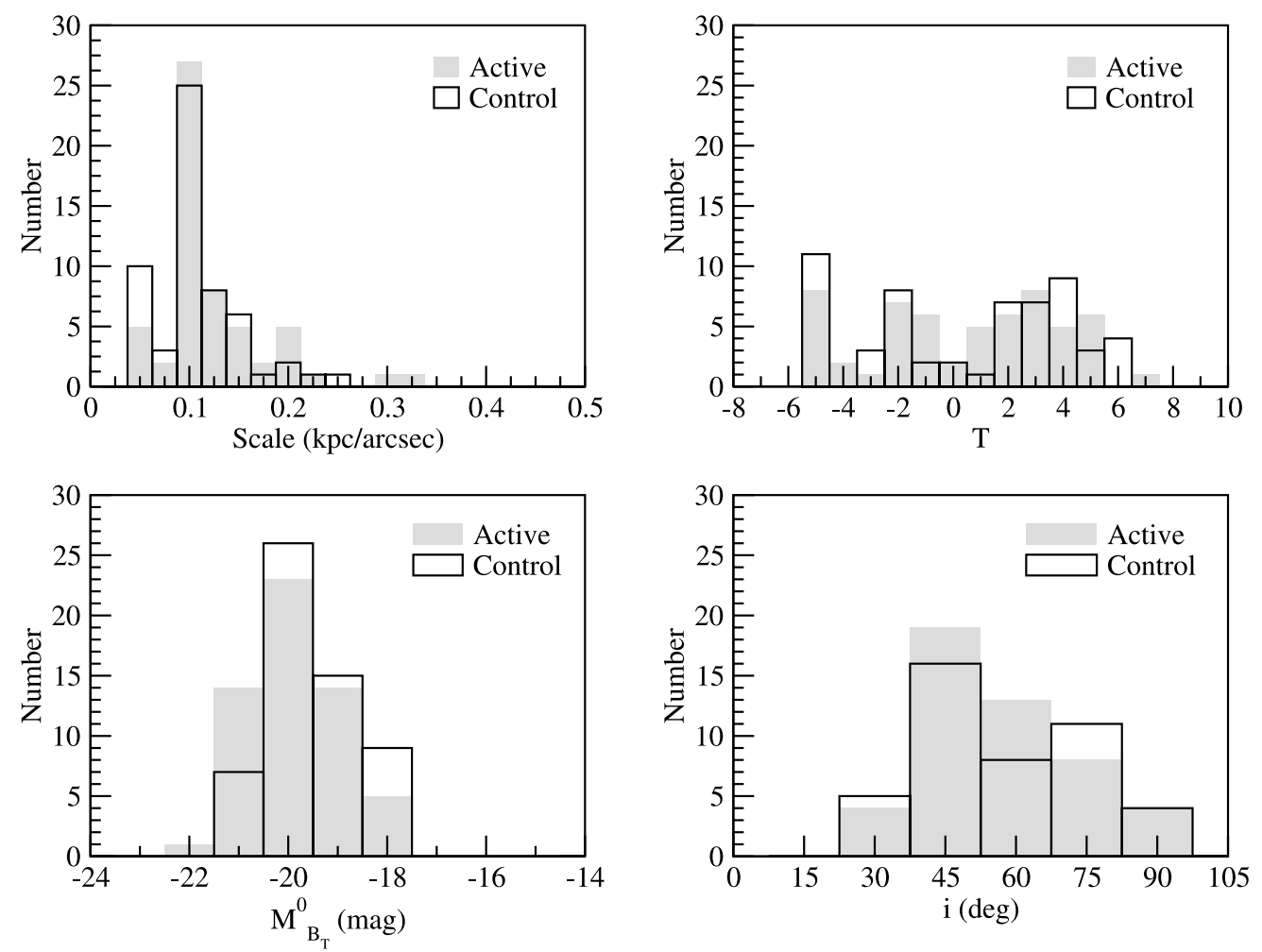

FIG. 1.-Distributions of plate scales, Hubble types, absolute $B$ magnitudes, and inclinations for all active and control galaxies of the matched sample.
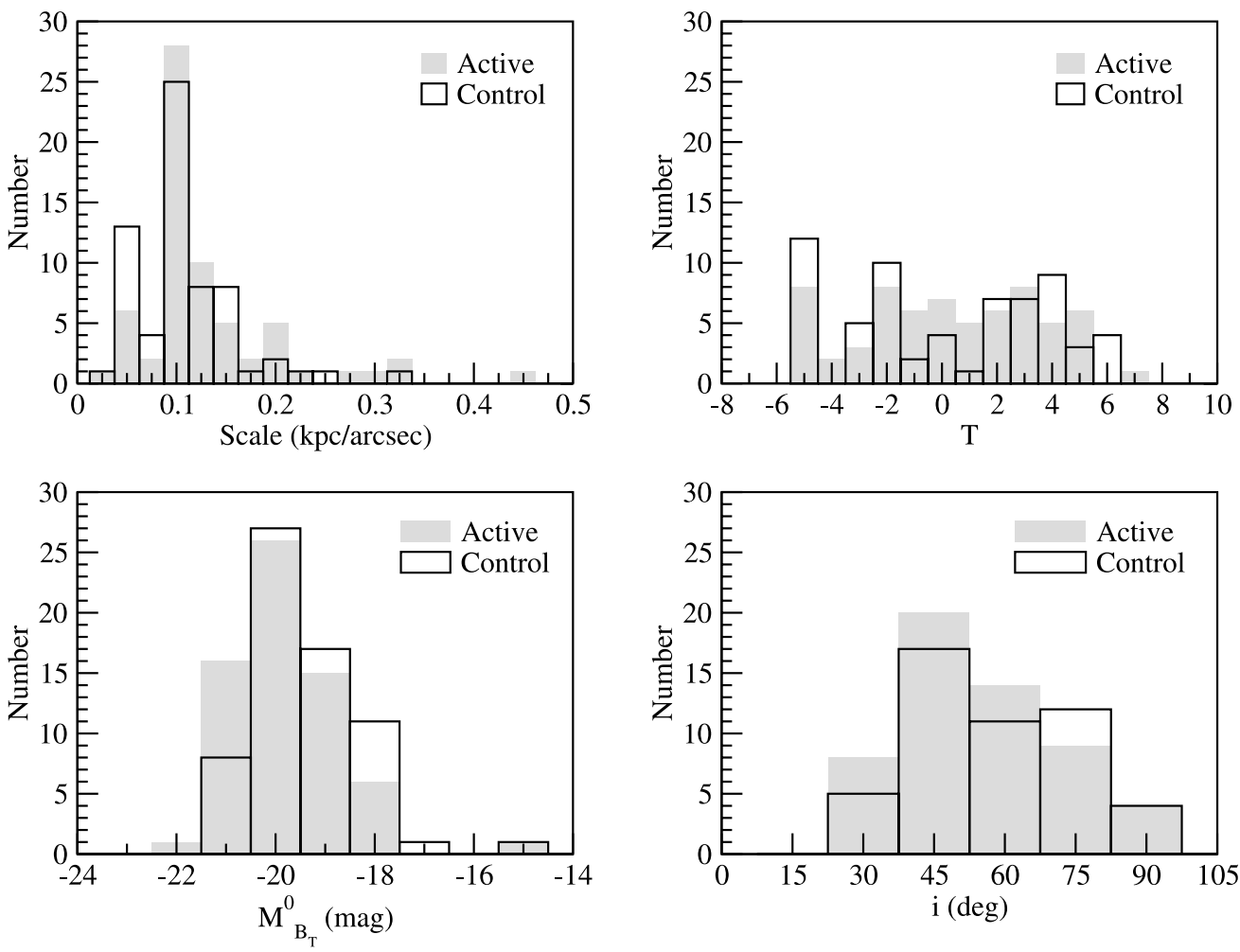

FIG. 2.-Distributions of plate scales, Hubble types, absolute $B$ magnitudes, and inclinations for all active and control galaxies of the extended sample. 
TABLE 1

hST Data and General Properties of the Active Sample

\begin{tabular}{|c|c|c|c|c|c|c|c|c|c|c|c|c|}
\hline $\begin{array}{c}\text { NGC Number } \\
\text { (1) }\end{array}$ & $\begin{array}{c}\text { WFPC } 2 \text { Camera } \\
\text { (2) }\end{array}$ & $\begin{array}{l}\text { Filter } \\
(3)\end{array}$ & $\begin{array}{c}\text { Exposure } \\
\text { (s) } \\
\text { (4) }\end{array}$ & $\begin{array}{c}T \\
(5)\end{array}$ & $\begin{array}{c}D \\
(\mathrm{Mpc}) \\
(6)\end{array}$ & $\begin{array}{c}M_{B_{T}}^{0} \\
(\mathrm{mag}) \\
(7)\end{array}$ & $\begin{array}{c}i \\
(\mathrm{deg}) \\
(8)\end{array}$ & $\begin{array}{l}\text { Activity Type } \\
\text { (9) }\end{array}$ & $\begin{array}{l}\text { Pair Number } \\
\quad(10)\end{array}$ & $\begin{array}{c}\text { Dust } \\
(\mathrm{kpc}) \\
(11)\end{array}$ & $\begin{array}{c}\text { Disk } \\
(\mathrm{kpc}) \\
(12)\end{array}$ & $\begin{array}{c}\text { Comments } \\
\text { (13) }\end{array}$ \\
\hline $0315 \ldots \ldots \ldots \ldots \ldots \ldots$. & $\mathrm{PC}$ & F555W & 230 & -4 & 65.8 & -22.22 & 52 & L1.9 & 7619 & 0.41 & $\ldots$ & $\mathrm{d}, \mathrm{i}$ \\
\hline $2655 \ldots \ldots \ldots \ldots \ldots . .$. & $\mathrm{PC}$ & F547M & 300 & 0 & 24.4 & -21.12 & 34 & $\mathrm{~S} 2$ & 4382 & $x$ & $\ldots$ & $\mathrm{i}, 1$ \\
\hline 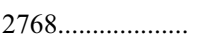 & $\mathrm{PC}$ & F555W & 233 & -5 & 23.7 & -21.17 & & L2 & 4406 & 0.64 & $\ldots$ & i, s \\
\hline $3226 \ldots \ldots \ldots \ldots \ldots \ldots$ & PC & F547M & 460 & -5 & 23.4 & -19.4 & $\ldots$ & L1.9 & 5576 & 0.36 & $\ldots$ & $\mathrm{d}$ \\
\hline 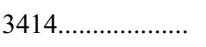 & $\mathrm{PC}$ & F555W & 160 & -2 & 24.9 & -20.12 & 44 & $\mathrm{~L} 2$ & 4371 & 0.15 & $\ldots$ & s \\
\hline $3516 \ldots \ldots \ldots \ldots \ldots \ldots . .$. & $\mathrm{PC}$ & F555W & 1000 & -2 & 38.9 & -20.81 & 40 & $\mathrm{~S} 1.2$ & 2950 & 0.9 & $\ldots$ & $\mathrm{i}, \mathrm{s}$ \\
\hline 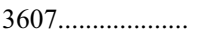 & $\mathrm{PC}$ & F547M & 260 & -2 & 19.9 & -20.7 & 62 & L2 & 4526 & 1.23 & $\ldots$ & $\mathrm{b} ?, \mathrm{~d}, \mathrm{~s}$ \\
\hline 3945 ...................... & $\mathrm{PC}$ & F555W & 600 & -1 & 22.5 & -20.41 & 50 & $\mathrm{~L} 2$ & 4621 & $\times$ & $\ldots$ & $\mathrm{i}$ \\
\hline 3998........................ & $\mathrm{PC}$ & F547M & 240 & -2 & 21.6 & -20.18 & 34 & L1.9 & 2300 & 0.43 & $\ldots$ & $\mathrm{i}, 1$ \\
\hline 4261..................... & $\mathrm{PC}$ & F547M & 800 & -5 & 35.1 & -21.37 & $\ldots$ & $\mathrm{L} 2$ & 3640 & 0.15 & $\ldots$ & d \\
\hline $4278 \ldots \ldots \ldots \ldots \ldots . .$. & $\mathrm{PC}$ & F555W & 1000 & -5 & 9.7 & -18.96 & 22.53 & L1.9 & 3377 & $x$ & $\ldots$ & i \\
\hline $4293 \ldots \ldots \ldots \ldots \ldots . .$. & WF & F606W & 160 & 0 & 17 & -20.23 & 65 & $\mathrm{~L} 2$ & 4469 & $\times$ & $\ldots$ & 1 \\
\hline 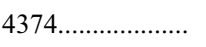 & PC & F547M & 1200 & -5 & 16.8 & -21.12 & $\ldots$ & L2 & 4649 & 0.52 & $\ldots$ & 1 \\
\hline $4486 \ldots \ldots \ldots \ldots \ldots \ldots . .$. & $\mathrm{PC}$ & F606W & 400 & -4 & 16.8 & -21.64 & $\ldots$ & L2 & 4365 & $\times$ & $\ldots$ & $\mathrm{i}$ \\
\hline $4550 \ldots \ldots \ldots \ldots \ldots \ldots . .$. & $\mathrm{PC}$ & F555W & 400 & -1.5 & 16.8 & -18.77 & 78 & L2 & 4570 & 0.77 & $\ldots$ & $\mathrm{i}, \mathrm{s}$ \\
\hline $4589 \ldots \ldots \ldots \ldots \ldots \ldots . .$. & $\mathrm{PC}$ & F555W & 1000 & -5 & 30 & -20.71 & $\ldots$ & L2 & 5557 & $x$ & $\ldots$ & $\mathrm{i}, 1$ \\
\hline $4636 \ldots \ldots \ldots \ldots \ldots . .$. & $\mathrm{PC}$ & F547M & 500 & -5 & 17 & -20.72 & $\ldots$ & L1.9 & 0821 & $x$ & $\ldots$ & $\mathrm{i}$ \\
\hline 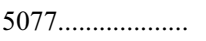 & $\mathrm{PC}$ & F547M & 260 & -5 & 40.6 & -20.83 & $\ldots$ & L1.9 & 4291 & $x$ & $\ldots$ & $\mathrm{w}$ \\
\hline 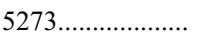 & $\mathrm{PC}$ & F606W & 560 & -2 & 21.3 & -19.26 & 24 & $\mathrm{~S} 1.5$ & 4379 & 0.14 & $\ldots$ & $1, \mathrm{~s}$ \\
\hline 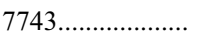 & $\mathrm{PC}$ & F606W & 560 & -1 & 24.4 & -19.78 & 32 & $\mathrm{~S} 2$ & 4578 & 0.73 & $\ldots$ & $\mathrm{s}$ \\
\hline
\end{tabular}

Early-Type Extended Sample

\begin{tabular}{|c|c|c|c|c|c|c|c|c|c|c|c|c|}
\hline$\ldots \ldots \ldots$ & $\mathrm{PC}$ & F606W & 280 & -3 & 2.4 & -15.98 & $\ldots$ & L2 & 0221 & 0.04 & $\ldots$ & $\mathrm{s}$ \\
\hline $1358 .$. & $\mathrm{PC}$ & F606W & 500 & 0 & 53.6 & -20.95 & 38 & $\mathrm{~S} 2$ & 7457 & 0.76 & $\ldots$ & $\mathrm{s}$ \\
\hline 3166.................... & $\mathrm{PC}$ & F547M & 300 & 0 & 22 & -20.7 & 62 & L2 & 3115 & 0.92 & $\ldots$ & $\mathrm{i}, 1, \mathrm{~s}$ \\
\hline $3884 \ldots \ldots$ & $\mathrm{PC}$ & F547M & 460 & 0 & 91.6 & -21.55 & 51 & L1.9 & 4405 & 0.77 & $\ldots$ & $\mathrm{s}$ \\
\hline 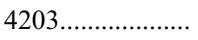 & $\mathrm{PC}$ & F555W & 160 & -3 & 9.7 & -18.32 & 21 & L1.9 & 4245 & 0.23 & $\ldots$ & $\mathrm{s}, 1$ \\
\hline $4477 \ldots \ldots \ldots \ldots \ldots \ldots$ & $\mathrm{PC}$ & F606W & 160 & -2 & 16.8 & -19.83 & 24 & $\mathrm{~S} 2$ & 3610 & 0.24 & $\ldots$ & $\mathrm{s}$ \\
\hline $5548 \ldots \ldots \ldots \ldots \ldots \ldots \ldots$ & $\mathrm{PC}$ & F606W & 500 & 0 & 67 & -21.32 & 27 & $\mathrm{~S} 1.5$ & 0507 & 2.54 & $\ldots$ & $\mathrm{s}$ \\
\hline $6340 \ldots \ldots \ldots \ldots \ldots \ldots$ & $\mathrm{PC}$ & F606W & 300 & 0 & 22 & -20.04 & 24 & L2 & 3412 & 0.03 & $\ldots$ & d \\
\hline
\end{tabular}

\begin{tabular}{|c|c|c|c|c|c|c|c|c|c|c|c|c|}
\hline \multicolumn{13}{|c|}{ Late-Type Matched Sample } \\
\hline $1058 \ldots$ & $\mathrm{PC}$ & F606W & 160 & 5 & 9.1 & -18.25 & 21 & $\mathrm{~S} 2$ & 3344 & $\times$ & $\ldots$ & $\ldots$ \\
\hline $1068 \ldots \ldots \ldots \ldots \ldots \ldots$ & $\mathrm{PC}$ & F606W & 560 & 3 & 14.4 & -21.32 & 32 & $\mathrm{~S} 1.8$ & 3310 & $x$ & $\ldots$ & $\ldots$ \\
\hline 1667.................... & $\mathrm{PC}$ & F606W & 500 & 5 & 61.2 & -21.52 & 40 & $\mathrm{~S} 2$ & 2339 & $\times$ & $\ldots$ & $\ldots$ \\
\hline $2273 \ldots \ldots \ldots \ldots \ldots \ldots$ & $\mathrm{PC}$ & F606W & 500 & 0.5 & 28.4 & -20.25 & 41 & $\mathrm{~S} 2$ & 2782 & $x$ & $\ldots$ & $\ldots$ \\
\hline $2639 \ldots \ldots \ldots \ldots \ldots \ldots$ & $\mathrm{PC}$ & F606W & 500 & 1 & 42.6 & -20.96 & 54 & S1.9 & 0972 & $\times$ & $\cdots$ & $\ldots$ \\
\hline $3079 \ldots \ldots \ldots \ldots \ldots \ldots$ & $\mathrm{PC}$ & F606W & 560 & 7 & 20.4 & -21.14 & 90 & $\mathrm{~S} 2$ & 3556 & $x$ & $\ldots$ & $\ldots$ \\
\hline $3368 \ldots \ldots \ldots \ldots \ldots \ldots$ & $\mathrm{PC}$ & F606W & 280 & 2 & 8.1 & -19.74 & 47 & L2 & 3351 & $\times$ & $\ldots$ & $\ldots$ \\
\hline $3486 \ldots \ldots \ldots \ldots \ldots \ldots$ & $\mathrm{PC}$ & F606W & 560 & 5 & 7.4 & -18.58 & 43 & $\mathrm{~S} 2$ & 3423 & $\times$ & $\ldots$ & $\ldots$ \\
\hline 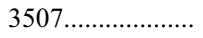 & $\mathrm{PC}$ & F606W & 80 & 3 & 19.8 & -19.86 & 32 & L2 & 4041 & $\times$ & $\ldots$ & $\ldots$ \\
\hline $3642 \ldots \ldots \ldots \ldots \ldots \ldots$ & $\mathrm{PC}$ & F547M & 300 & 4 & 27.5 & -20.74 & 34 & L1.9 & 5383 & $\times$ & $\ldots$ & $\ldots$ \\
\hline $3718 \ldots \ldots \ldots \ldots \ldots \ldots$ & $\mathrm{PC}$ & F547M & 300 & 1 & 17 & -19.96 & 62 & L1.9 & 4274 & $\times$ & $\ldots$ & $\ldots$ \\
\hline $3982 \ldots$ & $\mathrm{PC}$ & F606W & 500 & 3 & 17 & -19.47 & 30 & $\mathrm{~S} 1.9$ & 4800 & $\times$ & $\ldots$ & $\ldots$ \\
\hline $4051 \ldots \ldots \ldots \ldots \ldots \ldots$ & $\mathrm{PC}$ & F606W & 500 & 4 & 17 & -20.41 & 43 & $\mathrm{~S} 1.2$ & 6412 & $x$ & $\ldots$ & $\ldots$ \\
\hline $4258 \ldots \ldots \ldots \ldots \ldots \ldots$ & $\mathrm{PC}$ & F606W & 560 & 4 & 6.8 & -20.63 & 70 & S1.9 & 2903 & $x$ & $\ldots$ & $\ldots$ \\
\hline $4388 \ldots \ldots \ldots \ldots \ldots \ldots$ & $\mathrm{PC}$ & F606W & 560 & 3 & 16.8 & -20.34 & 83 & S1.9 & 4845 & $\times$ & $\ldots$ & $\ldots$ \\
\hline 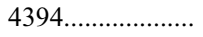 & WF & F606W & 320 & 3 & 16.8 & -19.62 & 27 & L2 & 0278 & $\times$ & $\ldots$ & $\ldots$ \\
\hline $4450 \ldots \ldots \ldots \ldots \ldots \ldots$ & $\mathrm{PC}$ & F555W & 520 & 2 & 16.8 & -20.38 & 43 & L1.9 & 4102 & $\times$ & $\ldots$ & $\ldots$ \\
\hline $4501 \ldots \ldots \ldots \ldots \ldots \ldots$ & $\mathrm{PC}$ & F606W & 600 & 3 & 16.8 & -21.27 & 59 & $\mathrm{~S} 2$ & 2748 & $x$ & $\ldots$ & $\ldots$ \\
\hline $4579 \ldots \ldots \ldots \ldots \ldots \ldots$ & $\mathrm{PC}$ & F547M & 726 & 3 & 16.8 & -20.84 & 38 & $\mathrm{~S} 1.9 / \mathrm{L} 1.9$ & 6217 & $\times$ & $\ldots$ & $\ldots$ \\
\hline $4639 \ldots \ldots \ldots \ldots \ldots \ldots$ & $\mathrm{PC}$ & F547M & 460 & 4 & 16.8 & -19.28 & 48 & $\mathrm{~S} 1.0$ & 0864 & $\times$ & $\ldots$ & $\ldots$ \\
\hline $4651 \ldots \ldots \ldots \ldots \ldots \ldots$ & $\mathrm{PC}$ & F555W & 660 & 5 & 16.8 & -20.09 & 50 & L2 & 4567 & $\times$ & $\ldots$ & $\ldots$ \\
\hline 4698..................... & $\mathrm{PC}$ & F606W & 600 & 2 & 16.8 & -19.89 & 53 & $\mathrm{~S} 2$ & 4380 & $\times$ & $\ldots$ & $\ldots$ \\
\hline
\end{tabular}


TABLE 1 - Continued

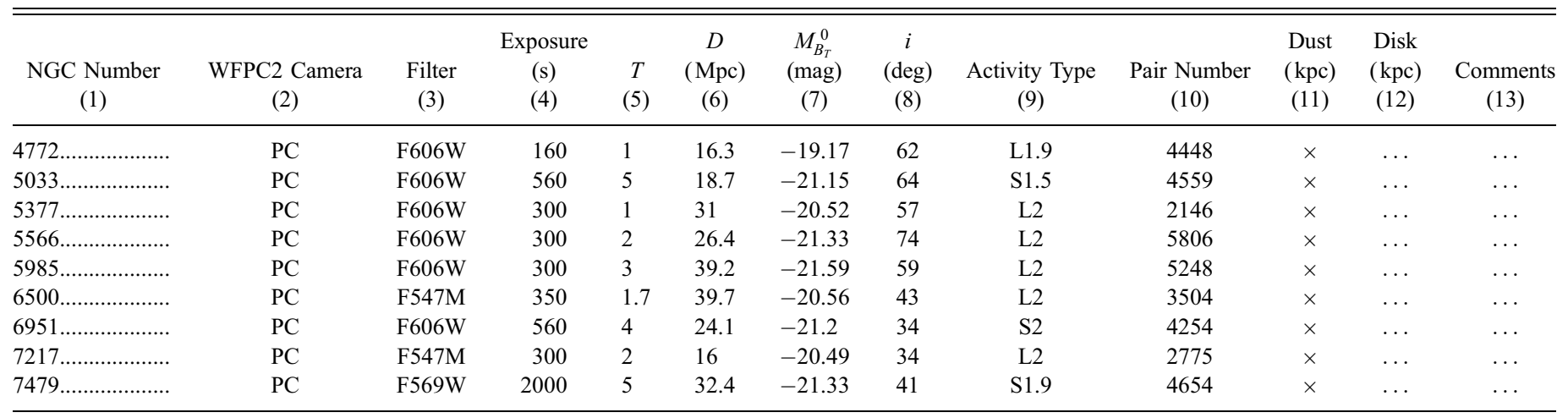

Notes.-Col. (3): Passband $H S T$ filter used in observation. Cols. (5)-(9): Galaxy type $T$; distance in Mpc; $B$-band absolute magnitude; inclination; and activity type from Ho et al. (1997a), where S indicates a Seyfert and L indicates a LINER. Col. (10) NGC number of the control sample pair. Col. (11) Main innermost dust structure semimajor axis size in kpc; a " $\times$ " sign means some dust structure is present but is difficult to measure. Col. (12) Stellar disk radial extent in kpc. Col. (13) Comments: (b): bar, (d): dust disk, (i): irregular dust, (l): dust lanes or filaments, (r): dust ring, (s): dust spiral, (w): weak feature, (?): uncertain classification.

Martini et al. (2003) investigate the incidence of dust in a wellmatched sample of 28 active and 28 inactive galaxies and found that all of the AGNs possess dust structure, while seven of the inactive galaxies did not.

Studies of the centers of early-type galaxies have also revealed that many contain disky isophotes, and in some cases these isophotes are most likely due to embedded stellar disks (e.g., Capaccioli et al. 1987; Bender et al. 1988; Kormendy \& Djorgovski 1989; Scorza \& Bender 1995; Seifert \& Scorza 1996). Observations with HST have identified many stellar disks within the central kiloparsec (hereafter nuclear stellar disks) that are distinct from the outer disks present in lenticular galaxies (van den Bosch et al. 1998; Scorza \& van den Bosch 1998; Morelli et al. 2004; Krajnović \& Jaffe 2004). With a large sample of 67 early-type galaxies, Rest et al. (2001) found nuclear stellar disks in as many as $51 \%$ of their sample, although many were misaligned and likely nuclear stellar bars. Given that disks are easiest to detect when nearly edge-on, the observed fraction is consistent with the presence of nuclear stellar disks in all early-type galaxies (Rix \& White 1990).

In the present paper we address the relation between circumnuclear dust structures and the nuclear activity in galaxies, with a particular emphasis on early-type galaxies. Unlike most previous work, we use a well-defined and homogeneous input sample, the Palomar sample of Ho et al. (1995), and have carefully drawn a pair-matched sample of 34 active and 34 inactive early-type galaxies (ellipticals and lenticulars), matched by the properties of their host galaxies, in order to obtain a robust measurement of the frequency of dust structures in active and inactive early-type galaxies. We have also identified and analyzed a pair-matched sample of 31 active and 31 inactive late-type galaxies. This paper is organized as follows: in $\S 2$ we present the sample selection and the pair-matching technique; in $\S 3$ we present data reduction and analysis procedures; in $\S \S 4$ and 5 we present our results and discussion, respectively; and finally in $\S 6$ we present our conclusions.

\section{SAMPLE}

A key issue in the comparison of active and inactive galaxies is the selection of a well-defined control sample known to be inactive. This is difficult, because identification of a low-luminosity AGN often requires less sensitive observations than does confirmation that a low-luminosity AGN is not present. In order to avoid this problem, we have drawn our sample from the large, uniform, and sensitive Palomar Survey of Ho et al. (1995). The Palomar
Survey contains spectra of the closest 486 bright galaxies in a set region of the sky and all of the nuclear spectra are classified as absorption-line nuclei, $\mathrm{H}$ II, LINERs, transition objects, and Seyfert galaxies (Ho et al. 1997a). The Palomar Survey is considered to be the most complete and homogeneous representation of the nearby universe. Our first selection of the active galaxy sample comprised all Palomar Seyfert and LINER galaxies with available broadband $H S T$ WFPC2 images in the optical spectral region, excluding transition objects and those with uncertain classification. The sample of inactive galaxies comprises all Palomar galaxies classified as absorption-line and $\mathrm{H}$ II nuclei with available broadband WFPC2 images in the optical spectral region.

We then carefully selected a well-matched control sample from this sample of inactive galaxies through the use of a pair-matching algorithm. This approach is similar to that adopted by Martini et al. (2003), who argue that the best way to match control samples is to identify a control galaxy pair for each active galaxy with similar values of all properties that may affect the identification of dust structure (e.g., morphology, distance, luminosity, inclination). More traditional and commonly employed techniques, in contrast, just match the mean or median value of each property between the target and control samples, or the distribution of each property individually, and may be susceptible to different correlations between various properties in the two samples.

In order to make a robust comparison between active and inactive galaxies, we have identified an inactive pair for each active galaxy with the following criteria: maximum difference between absolute magnitudes of $1 \mathrm{mag}$, maximum difference between morphological types $T$ of 1 , maximum difference between galaxy inclinations of $15^{\circ}$, and maximum difference in plate scale of $50 \%$. To accomplish this, we developed an algorithm that finds the largest possible number of pairs between the active and inactive galaxies given the above criteria. This yielded 26 earlytype and 31 late-type pairs. These 57 pairs comprise our main sample, and we shall hereafter refer to it as the "matched sample." In order to increase the early-type sample, we then reran the pairing algorithm with the remaining early-type galaxies after relaxing the matching constraints: maximum difference between absolute magnitudes of $3 \mathrm{mag}$, maximum difference between types $T$ of 3, maximum difference between galaxy inclinations of $35^{\circ}$, and maximum difference in plate scale of $80 \%$. This resulted in 8 additional early-type pairs for a total of 34 matched pairs of early-type galaxies. This sample, combined with our 31 late-type pairs, we shall refer to as the "extended sample." Histograms of the Hubble types, distances, inclinations, and 
TABLE 2

hSt Data and General Properties of Control Sample

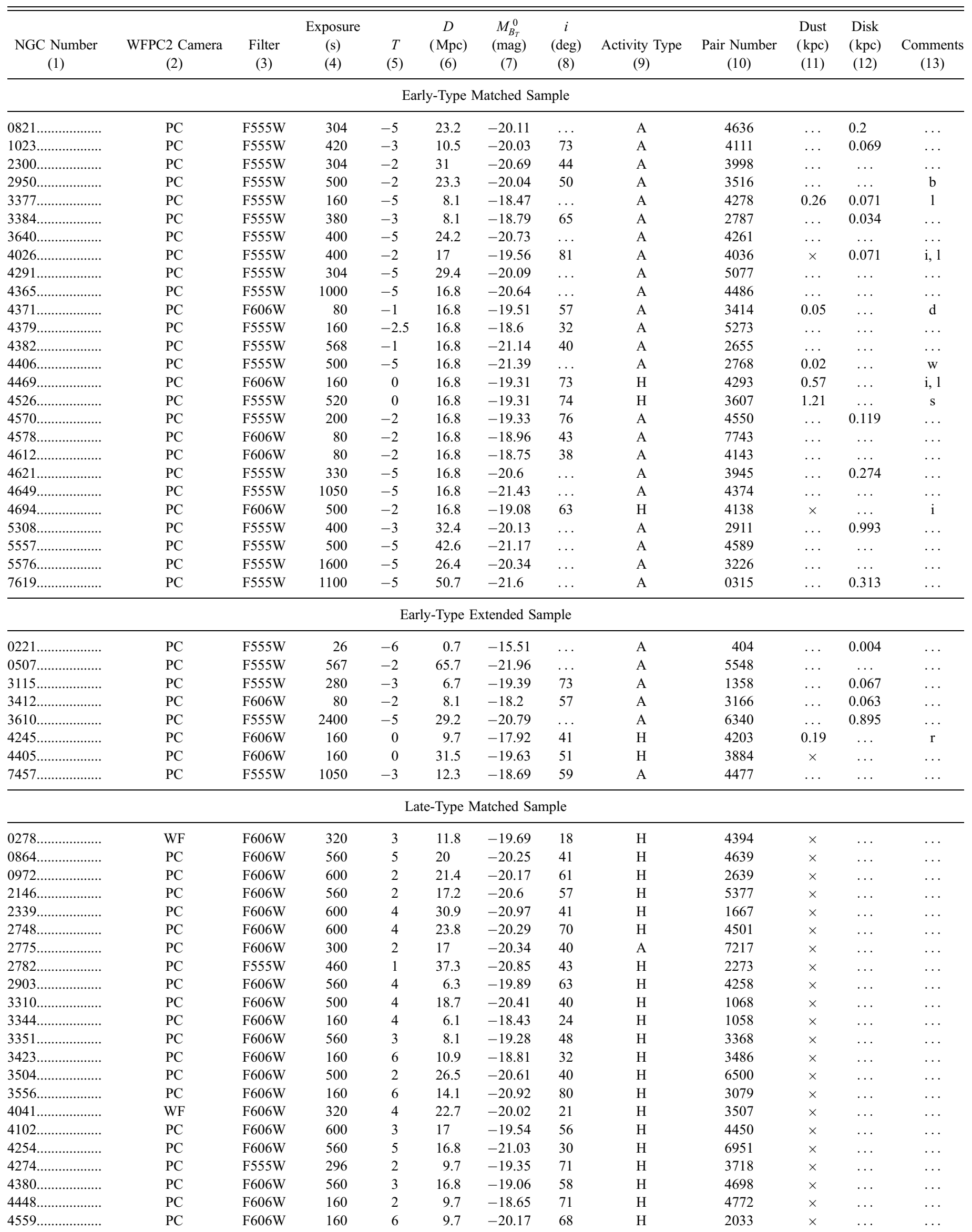


TABLE 2-Continued

\begin{tabular}{|c|c|c|c|c|c|c|c|c|c|c|c|c|}
\hline $\begin{array}{c}\text { NGC Number } \\
\text { (1) }\end{array}$ & $\begin{array}{c}\text { WFPC2 Camera } \\
\text { (2) }\end{array}$ & $\begin{array}{l}\text { Filter } \\
\text { (3) }\end{array}$ & $\begin{array}{c}\text { Exposure } \\
\text { (s) } \\
\text { (4) }\end{array}$ & $\begin{array}{c}T \\
(5)\end{array}$ & $\begin{array}{c}D \\
(\mathrm{Mpc}) \\
(6)\end{array}$ & $\begin{array}{c}M_{B_{T}}^{0} \\
(\mathrm{mag}) \\
(7)\end{array}$ & $\begin{array}{c}i \\
(\mathrm{deg}) \\
(8)\end{array}$ & $\begin{array}{c}\text { Activity Type } \\
\text { (9) }\end{array}$ & $\begin{array}{c}\text { Pair Number } \\
\text { (10) }\end{array}$ & $\begin{array}{c}\text { Dust } \\
(\mathrm{kpc}) \\
(11)\end{array}$ & $\begin{array}{c}\text { Disk } \\
(\mathrm{kpc}) \\
(12)\end{array}$ & $\begin{array}{c}\text { Comments } \\
\text { (13) }\end{array}$ \\
\hline 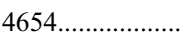 & $\mathrm{PC}$ & F606W & 160 & 6 & 16.8 & -20.38 & 56 & $\mathrm{H}$ & 7479 & $x$ & $\ldots$ & $\ldots$ \\
\hline $4800 \ldots \ldots \ldots \ldots \ldots \ldots$ & $\mathrm{PC}$ & F606W & 160 & 3 & 15.2 & -18.78 & 43 & $\mathrm{H}$ & 3982 & $x$ & $\ldots$ & $\ldots$ \\
\hline 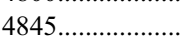 & $\mathrm{PC}$ & F606W & 160 & 2 & 15.6 & -19.55 & 79 & $\mathrm{H}$ & 4388 & $x$ & $\ldots$ & $\ldots$ \\
\hline 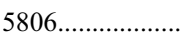 & $\mathrm{PC}$ & F606W & 600 & 3 & 28.5 & -20.43 & 61 & $\mathrm{H}$ & 5566 & $x$ & $\ldots$ & $\ldots$ \\
\hline 6217...................... & $\mathrm{PC}$ & F606W & 500 & 4 & 23.9 & -20.23 & 34 & $\mathrm{H}$ & 4579 & $x$ & $\ldots$ & $\ldots$ \\
\hline $6412 \ldots \ldots \ldots \ldots \ldots \ldots$ & $\mathrm{PC}$ & F606W & 560 & 5 & 23.5 & -19.78 & 30 & $\mathrm{H}$ & 4051 & $x$ & $\ldots$ & $\ldots$ \\
\hline
\end{tabular}

Notes.-Col. (3): Passband HST filter used in observation. Cols. (5)-(9): Galaxy type $T$; distance in Mpc; $B$-band absolute magnitude; inclination; and activity type from Ho et al. (1997a), where S indicates a Seyfert and L indicates a LINER. Col. (10) NGC number of the control sample pair. Col. (11) Main innermost dust structure semimajor axis size in kpc, a " $\times$ " sign means some dust structure is present but is difficult to measure. Col. (12) Stellar disk radial extent in kpc. (13) Comments: (b): bar, (d): dust disk, (i): irregular dust, (l): dust lanes or filaments, (r): dust ring, (s): dust spiral, (w): weak feature, (?): uncertain classification.

absolute $B$ magnitudes for the matched and extended sample are presented in Figures 1 and 2, respectively.

These figures show that there are similar distributions for each property in the active and inactive galaxies. Although this is expected for the matched sample, it also remains true for the extended sample and demonstrates that the additional 8 early-type galaxy pairs in the extended sample are reasonably well suited for comparison of the properties of active and inactive early-type galaxies. Relevant information for the sample galaxies is presented in Table 1, for the active galaxies, and Table 2, for the inactive galaxies. Columns (5)-(9) of each of these tables list the galaxy type $T$, distance, absolute magnitude, inclination, and activity type from Ho et al. (1997a).

\section{DATA REDUCTION AND ANALYSIS}

These data comprise HST WFPC2 images obtained both with the planetary and wide field cameras. Relevant data on the images, notably the camera, filter, and exposure times, are listed in columns (2)-(4) of Tables 1 and 2. All of the images were initially reduced by the $H S T$ archive's on-the-fly reprocessing pipeline. The only additional processing was cosmic-ray removal with the appropriate IRAF tasks. Specifically, when two or more images were available, we used the CRREJ task in the STSDAS package, otherwise we used the COSMICRAY task in the NOAO package.

To identify circumnuclear dust structure in these images, we used the structure map technique proposed by Pogge \& Martini (2002). Structure maps enhance structure as fine as the scale of the point-spread function (PSF) in an image and are well suited to the identification of narrow dust lanes and emission-line gas in nearby galaxies. The technique is based on the RichardsonLucy (R-L) image restoration algorithm, which uses the PSF to identify structure to enhance as part of the deconvolution process. Mathematically, a structure map $S$ is defined as

$$
S=[I /(I \otimes P)] P^{t},
$$

where $I$ is the original image, $P$ is the instrument PSF (constructed with the Tiny Tim software of Krist \& Hook 2004), $P^{t}$ is the transpose of the PSF, and $\otimes$ is the convolution operator (see Pogge \& Martini 2002).

The structure map technique recovers similar information to the more traditional methods of contrast enhancement, such as fitting and subtracting elliptical isophotes and construction of color maps, but offers several advantages. For example, the fitting and subtraction of ellipses can lead to artificial features in the presence of strong brightness discontinuities or isophotal twists in the images. In the case of color maps, there are simply not as many galaxies with observations in two filters as in one. Possible mismatches between the PSFs in the two bands could also introduce artificial features in color maps. These problems are all avoided with the use of structure maps, although some spurious features can still appear. For example, overexposed pixels can produce dark rings that mimic absorption features, while dead pixels can appear as false bright spots in the images. Nevertheless, such cases were easily identified and did not affect our analysis. After constructing structure maps, we then visually inspected each galaxy to determine if dust structures were present and measured the spatial extent of any dust. We also noted the presence of emission features that seem to be nuclear stellar disks (see discussion below) and measured the projected radial extent of these features.

\section{RESULTS}

Structure maps for all our galaxies are shown in Figures 3-5. Figure 3 presents structure maps of each early-type $(T \leq 0)$ AGN host next to its inactive control galaxy for the matched sample. Each galaxy pair has the active galaxy on the left and the inactive galaxy on the right. These two figures illustrate the most striking result of this paper, namely that all of the early-type active galaxies possess circumnuclear dust while only $27 \%$ ( 7 of 26) of the early-type inactive galaxies possess circumnuclear dust. Figure 4 demonstrates that this result also holds when we include the extended sample; specifically, all of the early-type active galaxies have circumnuclear dust and only $26 \%$ (9 of 34 ) of the inactive galaxies in the extended sample have circumnuclear dust. This result also holds if we exclude lenticulars and only consider ellipticals $(T=-5)$. Eight of eight (10 of 10) active ellipticals in our matched (extended) sample have dust, while only one of eight (2 of 15) inactive ellipticals in our matched (extended) sample have circumnuclear dust.

While previous investigators have noted an excess of circumnuclear dust in early-type active galaxies, compared to early-type inactive galaxies, ours is the first study to compare the frequency of circumnuclear dust between well-matched samples. In addition, both our active and inactive control samples have sensitive and uniform spectroscopy from the Palomar Survey. These data are important to confirm both the presence and absence of activity in the control sample. Some of the earliest evidence that earlytype active galaxies may more commonly possess dust came from studies of radio galaxies (Sadler \& Gerhard 1985; Marston 1988; Jaffe et al. 1994). Van Dokkum \& Franx (1995) presented 

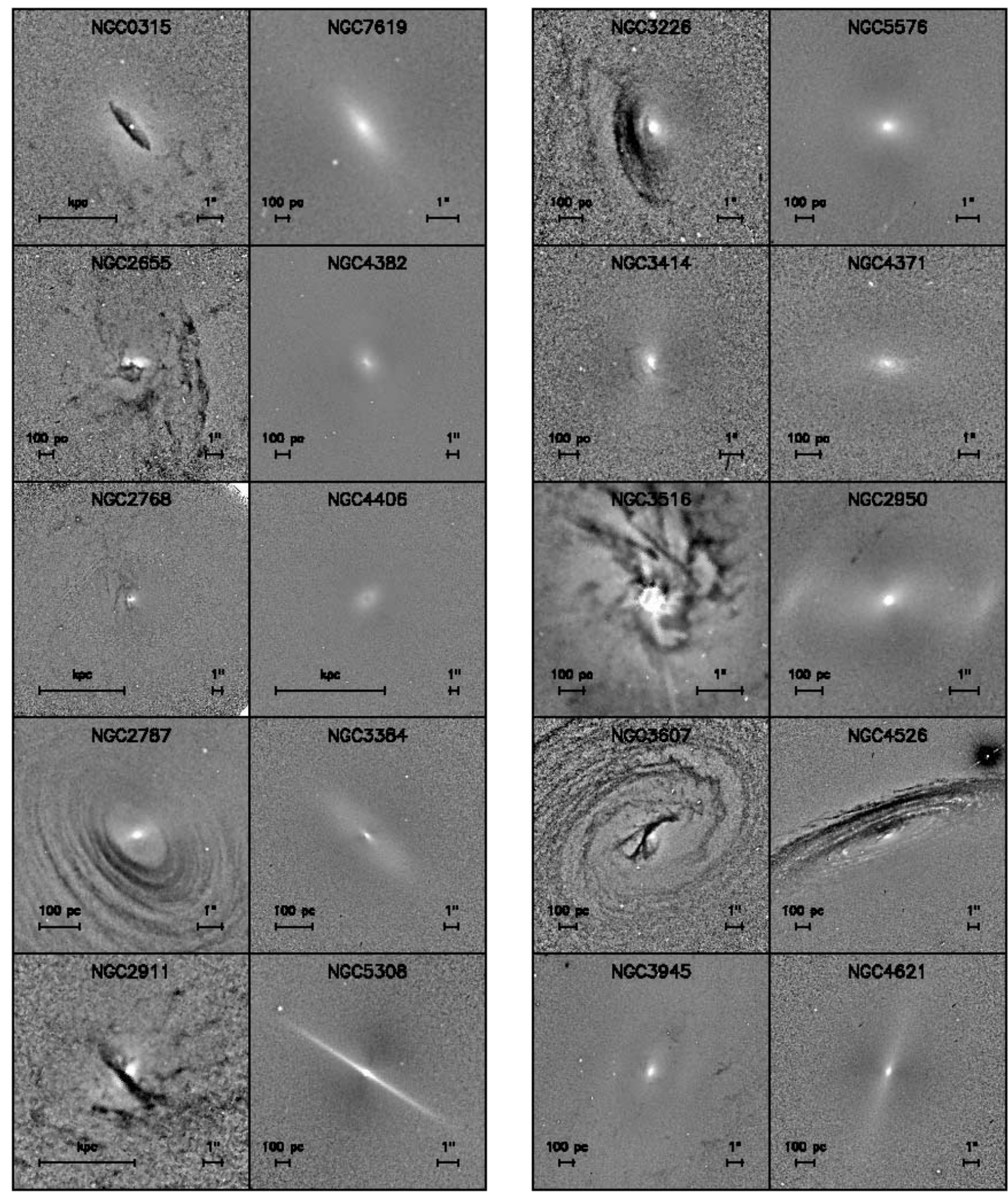

FIG. 3.-Structure maps for the matched sample of early-type galaxies. Each image covers 5\% of the galaxy D25 and is rotated so that north is up and east is to the left. In each panel, the left column displays the active galaxy and the right column the pair-matched control galaxy. 

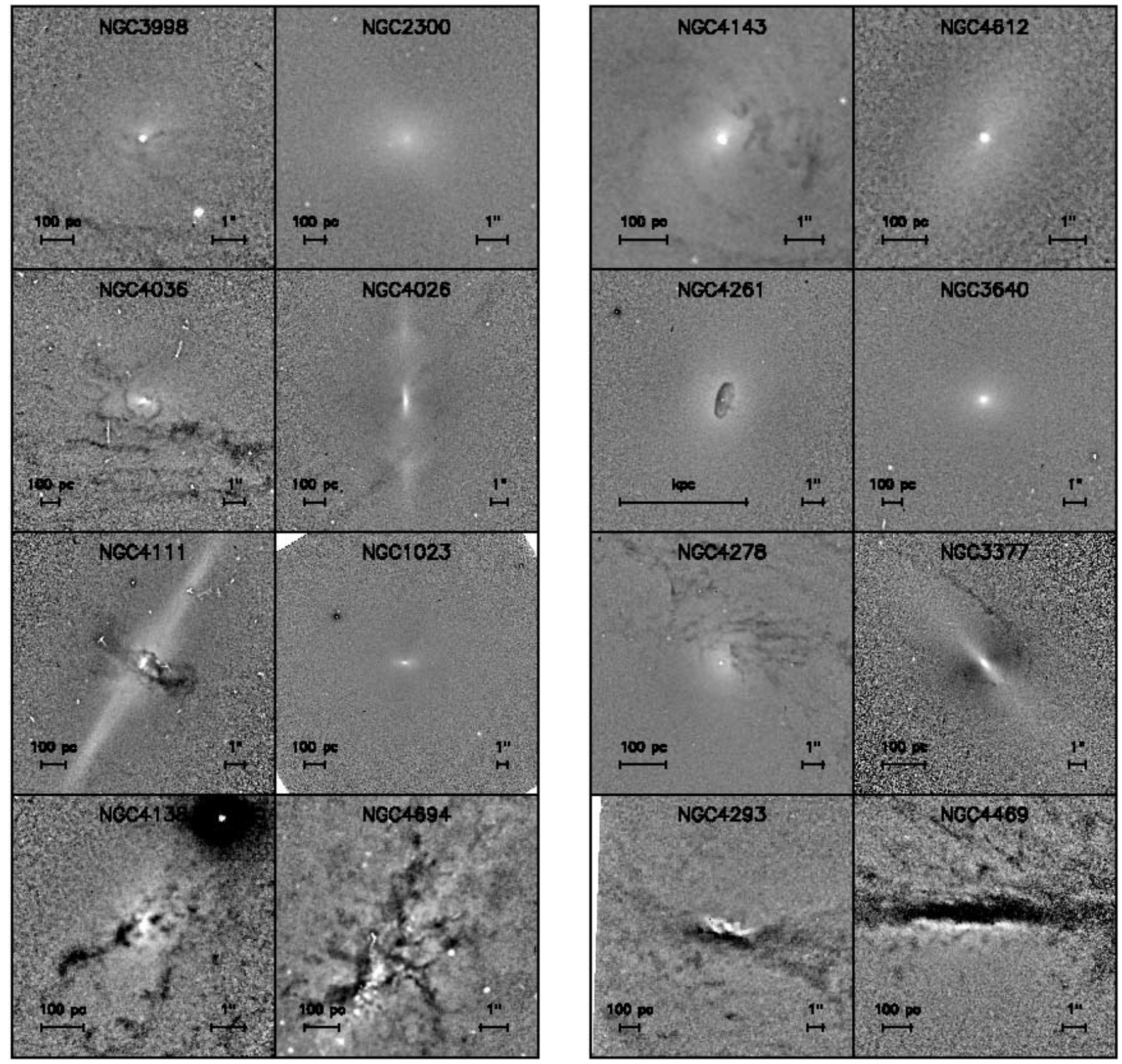

Fig. 3.-Continued

the first study of the incidence of dust in active and inactive early-type galaxies with $H S T$ and found dust in $72 \%$ of the radio galaxies, but only in 33\% of the galaxies without radio emission. Other investigators (Verdoes Kleijn et al. 1999; Ravindranath et al. 2001) have similarly found circumnuclear dust in most active galaxies, while they rarely found dust in inactive earlytype galaxies. The Ravindranath et al. (2001) sample is the most comparable to our own because that sample was also selected from the Palomar Survey, although their sample is somewhat smaller (33 galaxies total), did not match active and inactive hosts, and not all galaxies possessed visible-wavelength $H S T$ images. We attribute our $100 \%$ detection rate of dust in early-type AGN hosts to the excellent sensitivity of the Palomar Survey to lowluminosity AGNs.

The late-type $(T>0)$ galaxies are shown in Figure 5. All of these galaxies display evidence for circumnuclear dust regard- less of the presence or absence of detected nuclear activity. Previous studies of dust in galaxies with similar Hubble types have shown that circumnuclear dust is common in active late-type galaxies (e.g., Malkan et al. 1998; Regan \& Mulchaey 1999; Martini \& Pogge 1999; Pogge \& Martini 2002). Circumnuclear dust has also long been known to be quite common in late-type inactive galaxies. With a similarly matched sample of 28 active and 28 inactive galaxies, Martini et al. (2003) found that all active galaxies had circumnuclear dust, although $25 \%$ ( 7 of 28 ) of the inactive galaxies did not show evidence for circumnuclear dust. Only three of the seven inactive galaxies in their sample have Hubble type later than S0; therefore, their measured dust frequency is consistent with the presence of dust in all 31 of our late-type inactive sample. The frequency of circumnuclear dust structure as a function of Hubble type for the matched and extended samples is shown in Figure 6. 

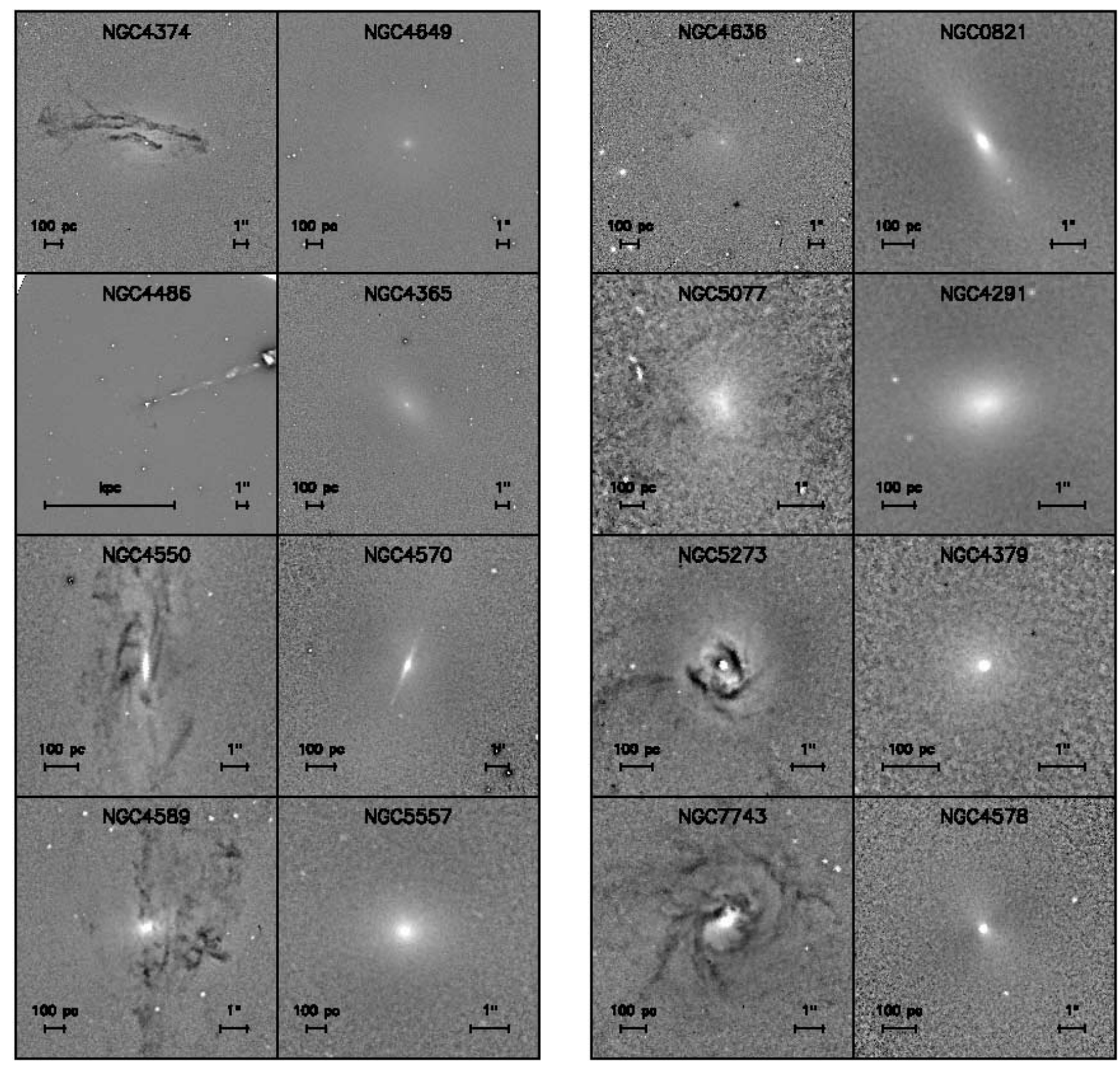

FIg. 3.-Continued

Most of the dust features in these early-type galaxies are dominated by dust lanes, and in many cases the lanes trace some spiral structure that indicates the presence of a dusty disk. In total, at least half of the active early-type galaxies exhibit some evidence for dust disks and $25 \%$ of these have tightly wound spirals in regular disks. These later dust structures are interesting because tightly wound dust structures are generally observed in the most axisymmetric (i.e., unbarred) late-type galaxies and suggest the presence of long-lived structures (Peeples \& Martini 2006). The presence of any dust structures in each galaxy is indicated in column (11) of Tables 1 and 2, where we report the projected radial extent of each dust feature in kiloparsecs. This radius was estimated by visual inspection of the digital images. A " $x$ " sign means that the radius of the structure is not clear and may extend beyond the borders of the image. For the late-type galaxies, we have not measured the radius of the dust structure as in most cases it does extend beyond the borders of the images. The dust disks and spirals have a mean size of $0.43 \pm 0.37$ and $0.69 \pm 0.63 \mathrm{kpc}$, respectively, for the early-type active galaxies. These structures therefore appear to be confined to the circumnuclear region or central kiloparsec, although these observations are less sensitive to dust on larger scales due to a decrease in signal-to-noise ratio. The position angles of the dusty disks in the early types, if apparent, are generally aligned with the position angle of the largescale isophotes. Some exceptions include orthogonal dust disks in NGC 2787, NGC 4111, and NGC 4143.

In addition to circumnuclear dust, our structure maps also reveal the presence of nuclear stellar disks in many galaxies. We have found disks in at least 13 of the 34 (38\%) early-type inactive galaxies, while we found nuclear stellar disks in only 1 of the 34 (3\%) early-type active galaxies. Many of these nuclear stellar disks were identified in previous work, such as NGC 4570, 

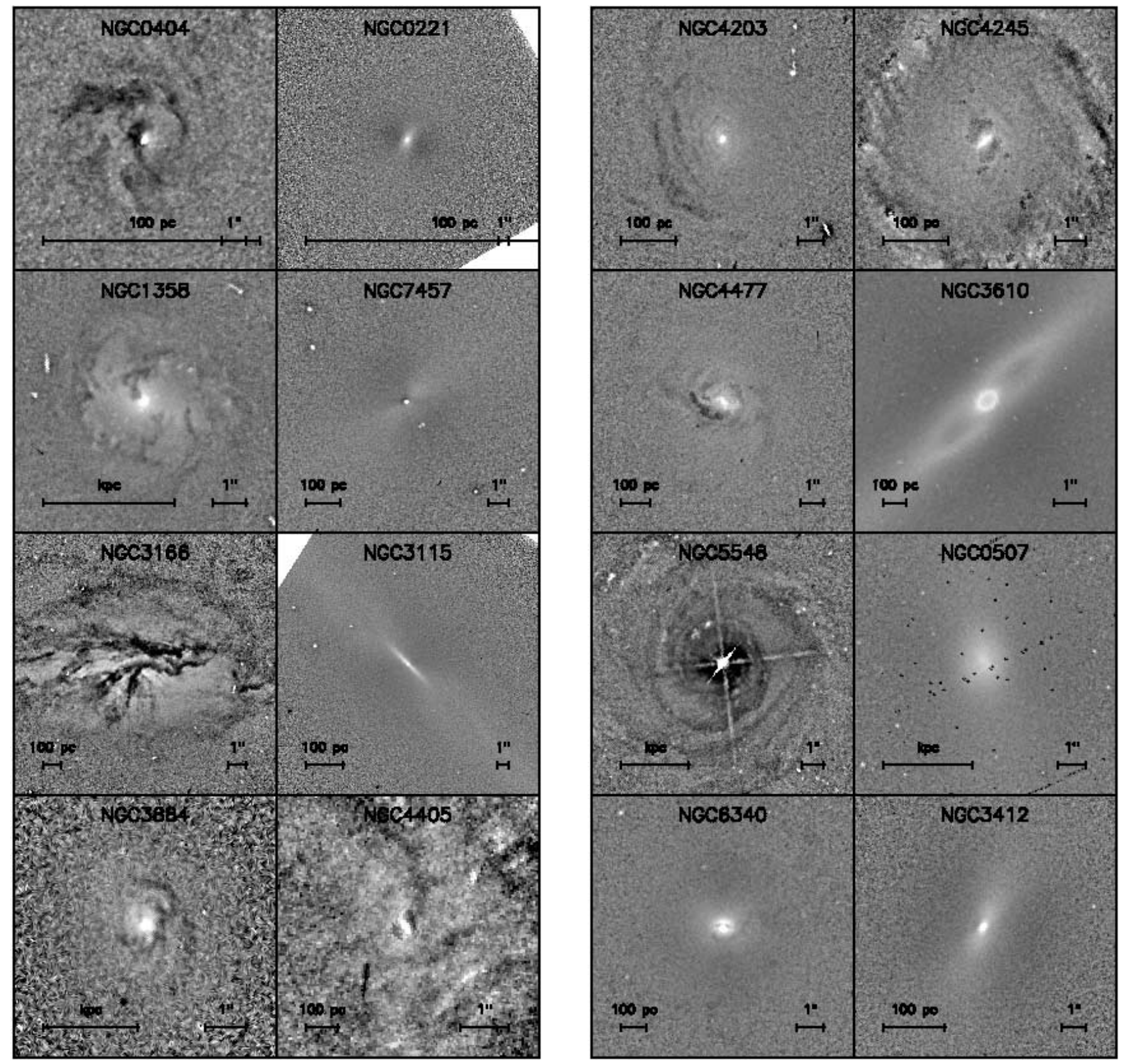

FIG. 4.- Structure maps for the extended sample of early-type galaxies. Images are presented as in Fig. 3.

NGC 4621, and NGC 5308 by Krajnović \& Jaffe (2004), which employed elliptical isophote fits to identify these disks. As we have identified a similar fraction of stellar disks, structure maps may be as sensitive to nuclear disks as isophote fitting. This is somewhat surprising, as structure maps are best suited to the detection of features on the scale of the PSF. The appearance of the stellar disks in the structure maps may therefore indicate that they are nearly edge-on, as is the case for elliptical isophote fits. Consistent with this interpretation, nearly all of the nuclear stellar disks in our sample have very large axis ratios. The detectability of stellar disks in early-type galaxies was modeled in detail by Rix \& White (1990) who found that the observed fraction is consistent with the presence of a nuclear stellar disk in every early-type galaxy.

The apparent nuclear stellar disks in these galaxies could also be nuclear stellar bars (e.g., Erwin \& Sparke 2002). To deter- mine if this is the case, we measured the position angle of each of the nuclear stellar disks and compared it with the position angle of the galaxy isophotes on large scales. In all cases the nuclear stellar disk was aligned with the position angle on larger scales, which suggests that they are not nuclear bars. Surprisingly, the position angles of the nuclear stellar disks in five out of five elliptical galaxies also agree with the position angle of the isophotes at large scales (NGC 821, NGC 3610, NGC 3377, NGC 4621, and NGC 7619). While some of these ellipticals are known to be disky (e.g., NGC 3377, NGC 4621), ellipticals are known to be triaxial and do not all rotate about their apparent minor axis (Ryden 1992). We return to this point in $\S 5.2$.

In contrast to the early-type inactive sample, we do not detect many nuclear stellar disks in the early-type active sample or in either of the late-type subsamples. The absence of nuclear disks in late-type galaxies has already been reported in the work of 

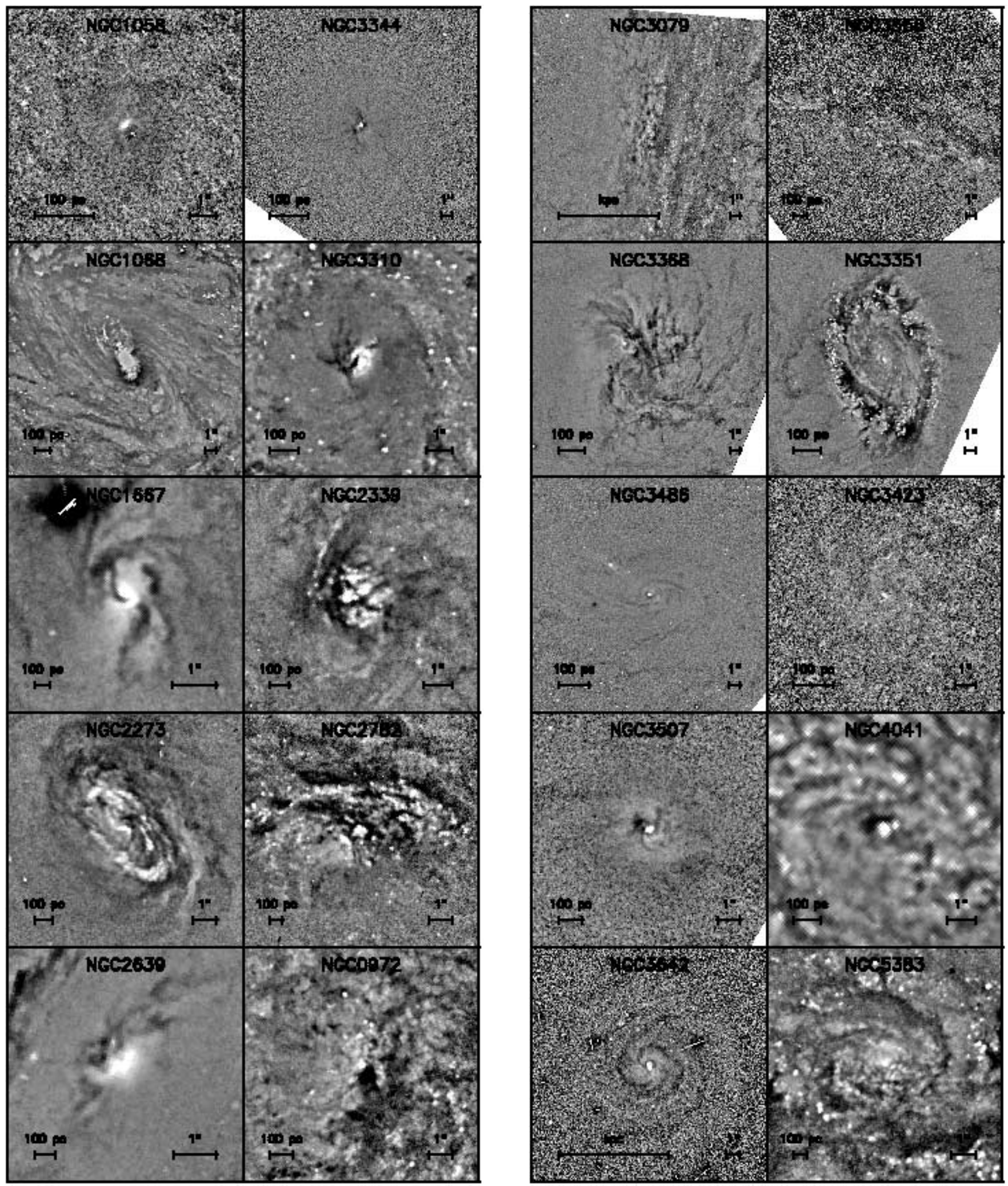

FIG. 5.-Structure maps for the matched sample of late-type galaxies. Images are presented as in Fig. 3.

Pizzella et al. (2002), who studied HST WFPC2 F606W images of a sample of 38 spiral galaxies and did not find nuclear disks in barred galaxies or galaxies of Hubble type later than $\mathrm{Sb}$. These differences from our early-type inactive sample are most likely due to the substantial circumnuclear dust in the early-type active sample and the late-type galaxies. In almost all cases, the dust (and emission-line regions) present in these galaxies make it difficult to identify nuclear stellar disks, particularly if the dust and stellar disks have the same position angle. Some notable exceptions are NGC 4111, which has a prominent stellar disk almost perpendicular to a smaller dust disk, and NGC 4026, which has a nuclear stellar disk and weak dust features. If nuclear stellar disks are also 

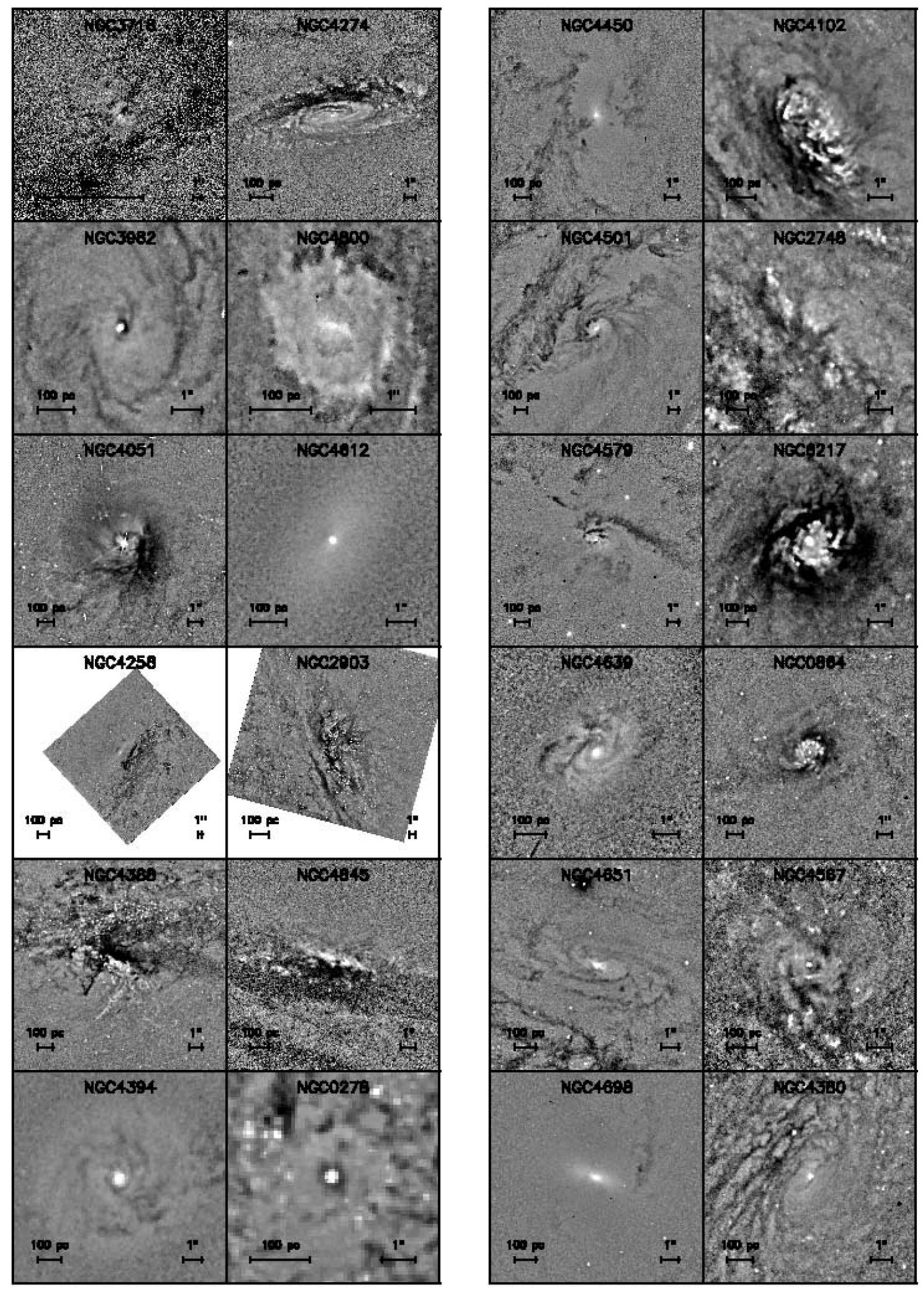

Fig. 5.-Continued 

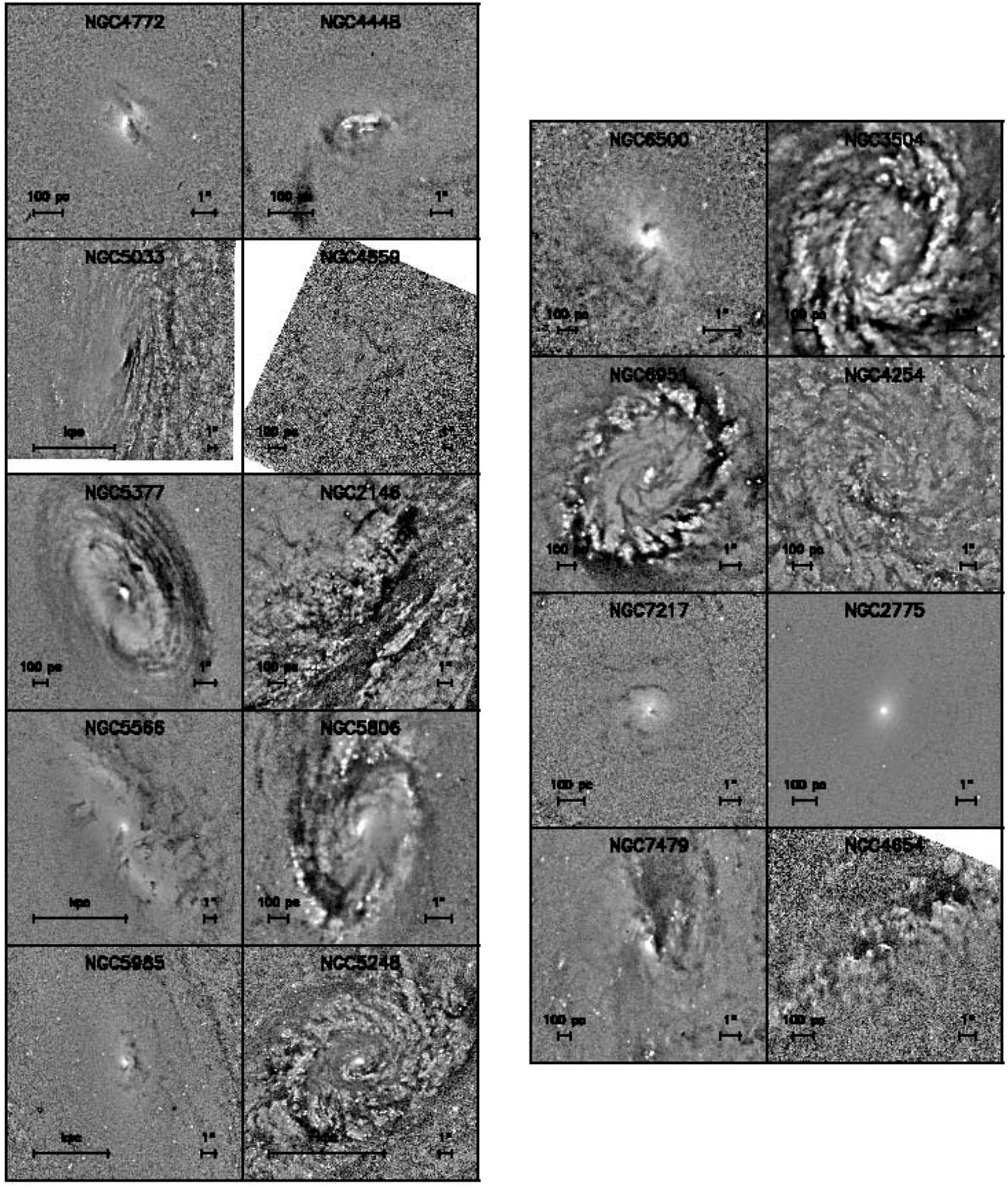

FIg. 5.-Continued

present in the dusty, active early types, the fraction may be higher in a study of near-infrared observations of early-type galaxies, although as Ravindranath et al. (2001) note the nuclear stellar disk fraction may also depend on the central surface brightness profile.

A histogram of the presence of stellar disks as a function of Hubble type in our matched and extended samples is shown in
Figure 7. The presence of nuclear stellar disks in our sample is identified in column (12) of Tables 1 and 2 and provides the radial extent of the structure in kiloparsecs. In Figure 8 we present a histogram showing the radial extent in kiloparsecs of the nuclear disks, which have a mean value of $0.24 \pm 0.31 \mathrm{kpc}$ and never extend beyond $1.1 \mathrm{kpc}$. Therefore, the bright stellar disks in the 

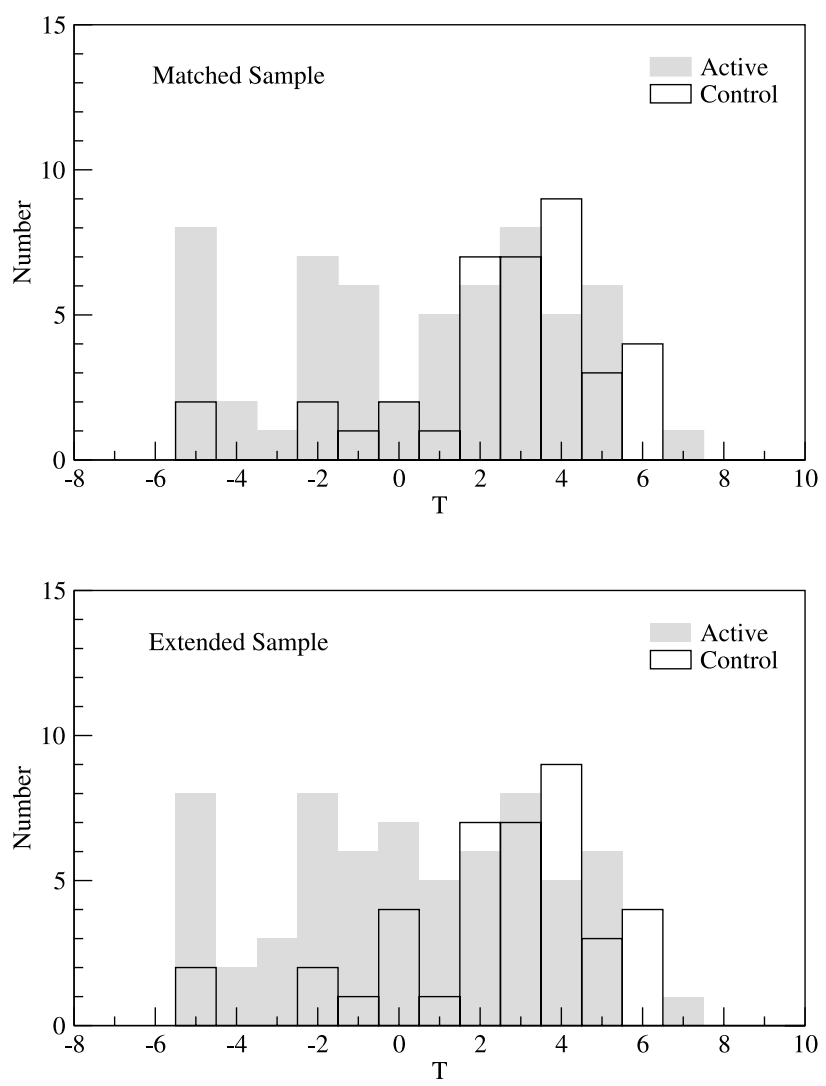

FIG. 6.- Presence of dust structure in the matched (top) and extended (bottom) sample as a function of Hubble type for the active and control galaxies.

inactive galaxies are nuclear structures, similar or somewhat smaller in average spatial extent to the dust structures observed in the active galaxies.

\section{DISCUSSION}

In $\S 4$ we identified two significant differences between active and inactive early-type galaxies. First, all active early-type galaxies have circumnuclear dust, while dust is only present in $26 \%$ of inactive early-type galaxies. Second, $38 \%$ of the inactive early-type galaxies have nuclear stellar disks, while they are detectable in almost none of the active early-type galaxies. In $\S \S 5.1$, 5.2 , and 5.3 we discuss these results in the context of AGN fueling, the origin of the dust and stellar disks, and present a simple evolutionary scenario for the centers of early-type galaxies.

\subsection{Ubiquitous Dust in Early-Type AGN Hosts}

Dust is present in all of the active early-type galaxies, in both the matched and extended samples. In contrast, dust was only found in $27 \%$ ( 7 of 26 ) of the inactive, matched sample and $26 \%$ (9 of 34) of the extended inactive sample. This strong correlation indicates that the presence of circumnuclear dust is a requirement for black hole accretion in early-type galaxies, while the presence of circumnuclear dust in some inactive galaxies indicates it is not a sufficient condition for activity. This strong correlation implies that the circumnuclear dust in these galaxies, most of which is hundreds of parsecs from the central black hole, is connected to or had a similar origin to the material currently fueling the active nucleus.

As the circumnuclear dust observed in early-type inactive galaxies almost always extends to the unresolved nucleus (tens of parsecs), this suggests that when dust is present in early-type
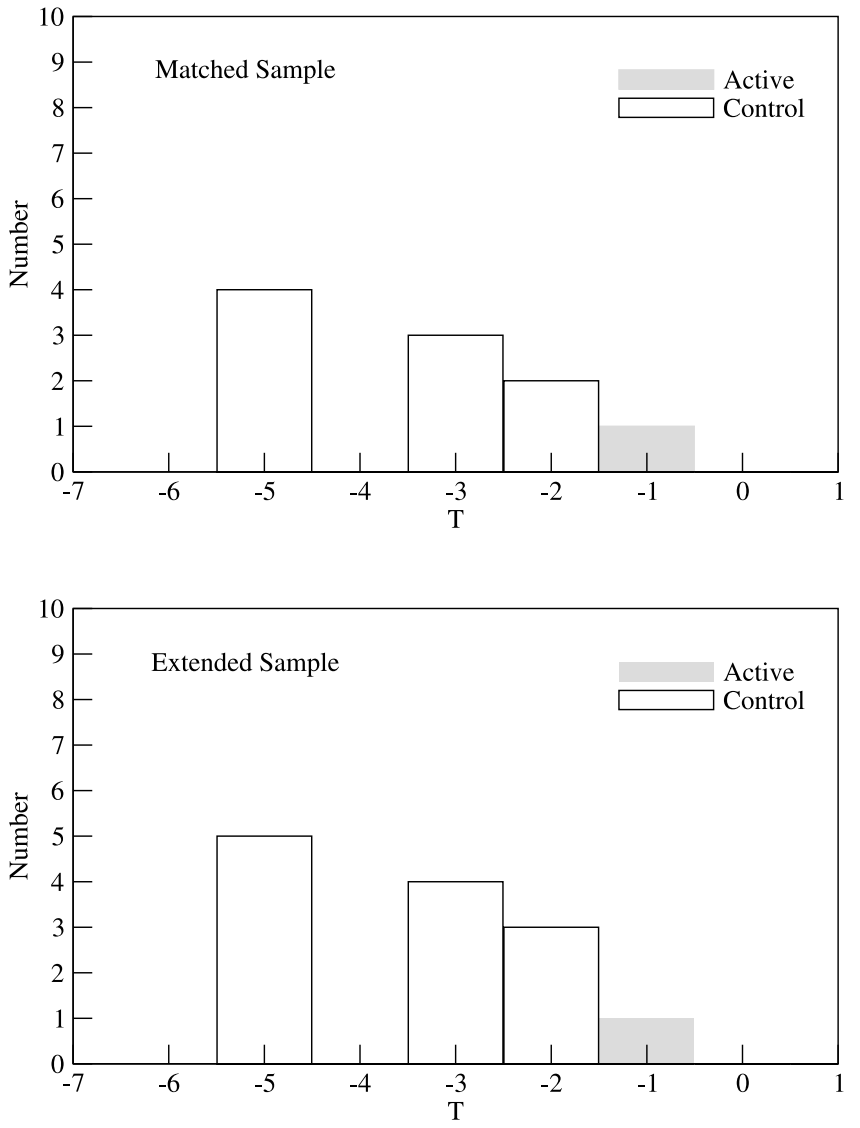

FIG. 7.-Presence of stellar disks in the matched (top) and extended (bottom) sample as a function of Hubble type for the active and control galaxies.

galaxies, at least some dust reaches the nucleus relatively quickly compared to its destruction time. The ratio of inactive to all earlytype galaxies with circumnuclear dust in a representative (as opposed to matched) sample multiplied by the dust lifetime could provide an estimate of how long it takes circumnuclear fuel to reach the nucleus. If we take the early-type galaxies in the Palomar Survey as representative of all early types, Ho et al. (1997b) found that approximately $50 \%$ of all early-type galaxies are AGNs. Our matched sample of early-type active and inactive galaxies is therefore likely representative of all early-type galaxies, and we infer that the time for some fuel to accrete onto the central black hole is approximately $20 \%$ of the lifetime that dust is observable in the circumnuclear region. This percentage would be yet smaller if the dust in the circumnuclear region compromised the identification of some early-type galaxies as active.

There are differing models for the origin and lifetime of dust in early-type galaxies. Unlike in late-type disks, there is not an obvious reservoir of dust on larger scales. The absence of such a dust reservoir indicates that the dust must be either created in situ or originate from outside of the galaxy. Stellar mass loss could produce the observed dust in early-type galaxies (Knapp et al. 1992). Athey et al. (2002) show that the mid-infrared dust emission from early-type galaxies is consistent with the expected stellar massloss rates, where the mass-loss rate is on order $1 M_{\odot} \mathrm{yr}^{-1}$ for a giant elliptical galaxy. The two difficulties with this process are that the dust should be produced in a uniform distribution that traces the stellar kinematics and that the dust will gradually be destroyed via sputtering by the hot gas that pervades these galaxies. The dust distribution in a flattened disk is somewhat puzzling in the elliptical galaxies, such as NGC 4261, because 

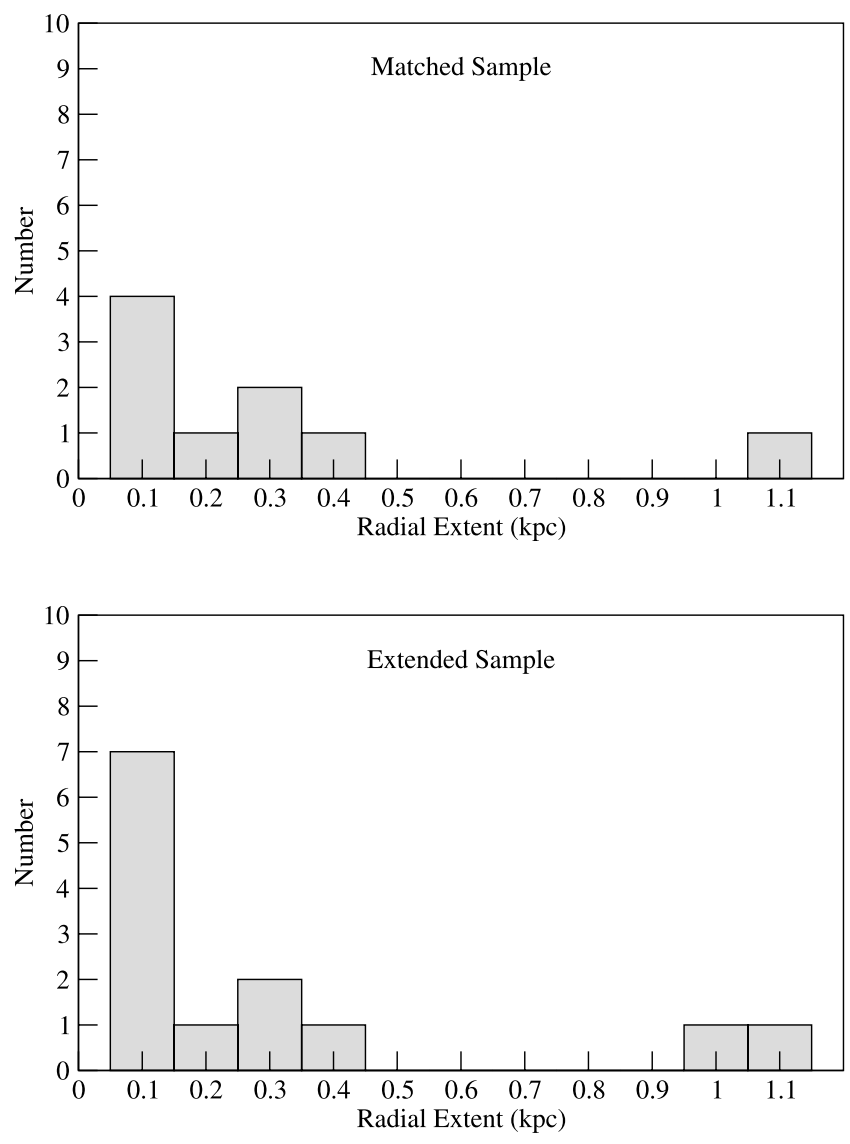

FIG. 8.- Stellar disk's radial extent in kpc for the matched (top) and extended (bottom) sample.

they generally do not exhibit substantial rotation, although even initially slow rotation would lead to substantial rotation if the dust settled into the central kiloparsec from large scales. Mathews \& Brighenti (2003) model the evolution of the dusty gas originating in stellar winds and found that cooling dust-rich gas could produce the observed circumnuclear dust, and in particular the observed clumping. However, even if dust cooling explains the observed clumpiness, stellar mass loss still cannot explain why circumnuclear dust is only present in $\sim 50 \%$ of all early-type galaxies. While dust destruction via collisions with hot gas will occur in on order of $10^{8} \mathrm{yr}$ (e.g., Mathews \& Brighenti 2003), mass loss will continually supply new dust to the interstellar medium. This difficulty with internal dust production is also reflected in the absence of a correlation between far-infrared and visible-wavelength luminosities in early-type galaxies, which also suggests the dust may have an external origin (Temi et al. 2004).

Kinematic observations of neutral and ionized gas in early-type galaxies have led many authors to conclude that this material has an external origin because the gas kinematics differ from the stellar kinematics (e.g., Bertola et al. 1984, 1992; Sarzi et al. 2006; Morganti et al. 2006, and references therein). The circumnuclear morphology of the dust also provides some additional constraints on the origin of the dust. In particular, the chaotic appearance suggests an external origin because internally created dust might be too uniformly distributed, particularly in lenticulars, if the cooling time discussed above is not shorter than the dynamical time. However, the circumnuclear dust observed in latetype galaxies exhibits a similar range in morphologies and that material is generally not held to have an external origin. The morphology of the dust can also provide some constraints on the lifetime of the dust. Lauer et al. (2005) discuss how the different dust morphologies can be viewed as a "settling sequence," where dust that is not in a disk must settle toward the center of the galaxy in a dynamical time, or a few $10^{7} \mathrm{yr}$ (see also Tran et al. 2001; Verdoes Kleijn \& de Zeeuw 2005), while coherent, tightly wound dust disks must be at least a few dynamical times old. The age of such a dust disk would then be set by the dust destruction timescale mentioned above, or $\sim 10^{8} \mathrm{yr}$. External dust input and a finite dust lifetime can plausibly explain why dust is not found in the centers of all early-type galaxies.

More broadly, the relative number of galaxies with chaotic dust lanes, some nuclear dust spirals, and very coherent dust disks could provide constraints on the settling time of the dust if there is a natural progression toward more ordered dust as the dust settles toward the galaxy nucleus or is destroyed. If this model is correct, then the dust in inactive galaxies should be most chaotic or unsettled. Our data are consistent with this interpretation, with the exception of the tightly wound dust spiral in NGC 4526, although there are only a small number of tightly wound dust spirals in our full early-type galaxy sample. In fact, the relative scarcity of the tightly wound dust spirals presents a problem because the estimates of the dust settling time suggest that these structures should be longer lived than the more commonly observed amorphous dust. As noted by Lauer et al. (2005), this suggests the total dust lifetime must be close to the dynamical time of several $\times 10^{7} \mathrm{yr}$, and these structures cannot be long lived.

Both the internal and external dust models rely on a dust destruction timescale of at most $10^{8} \mathrm{yr}$, set by sputtering, while the observed fraction of dust and AGNs in early-type galaxies indicates that about $50 \%$ of early-type galaxies have dust at any time, and this fraction approximately corresponds to the fraction with AGNs. The main problem with a purely internal origin is that the dust creation from mass loss and the destruction from sputtering are continuous processes and all early-type galaxies should have some dust. The internal origin model therefore requires some more cataclysmic event to destroy the dust on approximately the same timescale required to reform the observed dust mass. In this case, the central AGN provides the most ready supply of the necessary energy to unbind or destroy the dust. The external origin, in contrast, requires continual dust replenishment via mergers with gas and dust-rich galaxies. Given the high fraction of early-type galaxies with dust, the frequency of these mergers must be comparable to the dust destruction time. In this case, the estimate of $10^{8} \mathrm{yr}$ appears to require an incredibly high merger rate with gas-rich dwarf galaxies. If this is the case, the dusty or active early-type galaxies may preferentially be in richer environments or have more companions. We checked this with measurements of the average local galaxy density and average projected angular separation to the nearest neighbor from Ho et al. (1997a) and did not find evidence for higher local density or closer companions in the active or dusty samples.

\subsection{Nuclear Stellar Disks in Early-Type Galaxies}

Approximately a third of the inactive early-type galaxies have structures that appear to be nuclear stellar disks. As noted in $\S 4$, these are likely to be stellar disks, rather than nuclear stellar bars, because they have the same position angle as their host galaxy. The true incidence of stellar disks in our sample is likely to be higher than observed and may be $100 \%$, because most of these disks have very high axis ratios and we are most sensitive to stellar disks seen edge-on. Many previous studies have found nuclear stellar disks in the central regions of elliptical and lenticular 
galaxies. Ravindranath et al. (2001) found such disks in $21 \%$ of their 33 early-type galaxies, Lauer et al. (2005) found that nearly all of the power-law galaxies in their sample have stellar disks, and Ferrarese et al. (2006) find stellar disks in 13\% of a sample of 100 early-type galaxies in the Virgo Cluster. Nuclear stellar disks are therefore a common structure, although only a few have been studied in great detail (Pizzella et al. 2002; Morelli et al. 2004; Kormendy et al. 2005).

The origin of nuclear stellar disks has been a matter of recent debate, as these disks are not simple extensions of the large-scale disks to the centers of the galaxies. Krajnović \& Jaffe (2004) argue that the nuclear disks could be the result of the infall of mass to the center of the galaxy driven by the secular evolution of a bar, galaxy mergers, or both. These authors also point out that none of the previously investigated nuclear stellar disk galaxies has an active nucleus, in agreement with our findings (although they do harbor a $10^{8}-10^{9} M_{\odot} \mathrm{SMBH}$ ), and argue that this makes them descendants of quasars that spent their fuel and turned off the central engine. Some known nuclear disks do exhibit strong evidence for an external origin, such as that in NGC 4698 (Pizzella et al. 2002), which is geometrically decoupled from the host galaxy.

The ellipticity distribution of the five elliptical host galaxies with nuclear stellar disks may also have implications for the origin and detectability of these disks. As noted above, four of the five elliptical galaxies with nuclear stellar disks have very large axis ratios (three are classified E5 and one is classified E6?). Although this is a small sample, such large axis ratios are rare and suggests that nuclear stellar disks could preferentially exist in the subset of intrinsically flattened ellipticals and not just observed in the subset of the nuclear disks with a favorable viewing angle. As lower-luminosity ellipticals are more likely to be oblate or flattened (Vincent \& Ryden 2005) and tend to be faster rotators (Cappellari et al. 2006), the incidence of nuclear stellar disks should be reinvestigated as a function of elliptical galaxy properties, such as luminosity.

\subsection{Evolution and Timescales}

The nuclear stellar disks observed in early-type, inactive galaxies may share a common origin with the dusty disks observed in early-type AGN hosts. Once a nuclear stellar disk is formed in a galaxy, it provides a natural preferred plane of rotation for new cold gas and dust. New material, either infalling or formed in situ, would likely settle onto the disk in several rotation periods. While the dust settles, it would likely have a relatively chaotic appearance at first, although eventually it would form a relatively regular disk of dust and may host star formation that would increase the mass of the stellar disk. Kormendy et al. (2005) observe cospatial nuclear stellar and dust disks in the dwarf elliptical NGC 4486A and suggest that these disks are toward the end stage of this evolutionary scenario; namely, the observed stellar disk is forming from accreted gas and dust. This scenario is also supported by the recent study of Ferrarese et al. (2006). These authors find evidence of recent star formation associated with dusty disks but not with the irregular, unsettled dust lanes. They suggest a similar evolutionary scenario for dust settling in which dust is acquired externally, form large-scale dust lanes that gradually evolve to dusty disks with associated star formation, and then finally collapse to nuclear dusty disks associated with stellar disks. As star formation ceases and their colors become similar to the host galaxy, the disks then become more difficult to observe.

Additional support for the evolutionary scenario above is the kinematic evidence for inflow along nuclear spirals in at least one case: NGC 1097. Its nuclear dusty spiral has been revealed by near-IR observations (Prieto et al. 2005) and by structure maps in Fathi et al. (2006). Using the Integral Field Unit of the Gemini Multi-Object Spectrograph, the latter authors have found streaming motions along the nuclear spiral arms with inward velocities of up to $50 \mathrm{~km} \mathrm{~s}^{-1}$ in the $\mathrm{H} \alpha$ emitting gas throughout the nuclear region. Another relevant finding for this galaxy is the young obscured starburst discovered by Storchi-Bergmann et al. (2005) very close to the nucleus (within $\sim 10 \mathrm{pc}$ ), in agreement with the suggestion that inflowing gas and dust gives birth to stars in the nuclear dusty spiral or disk. A key point in testing this evolutionary scenario is the evaluation of the life cycles of the dusty and stellar disks. In the case of NGC 1097, the velocity observed for the streaming motions along the nuclear spiral allows an estimate of a few Myr for the gas to flow from a few hundred parsecs to the nucleus. This is also consistent with the above estimates that the dust may survive for on the order of several dynamical timescales.

If substantial star formation does add to stellar nuclear disks each activity cycle, then there should be some stars associated with the disks younger than the characteristic or episodic timescale of the dust replenishment and AGN lifetime, or younger than $10^{8} \mathrm{yr}$. While we do not have color information for our sample, a number of stellar disks are indeed found to be blue (Krajnović \& Jaffe 2004), although many authors also argue that the colors are the same as those of the bulge (Erwin \& Sparke 2002). Relatively old ages, from 6 to $15 \mathrm{Gyr}$, have been reported from studies using broadband colors and spectral indices (Krajnović \& Jaffe 2004; Morelli et al. 2004). Nevertheless, a close look at these studies reveals that the methods used to date the disk stellar population are not very sensitive to the presence of a small young component in the middle of a luminous old bulge. In particular, the spectral indices are measured in a restricted interval $(5000<\lambda<5400 \AA)$ and this wavelength range is not very sensitive to a small population of young stars and a blue spectral index should be used instead. The same applies for the broadband colors, which usually do not include images in the blue. Estimates of the total cold gas mass also suggest that the number of new stars formed from the observed dust is likely to be small compared to the mass of the stellar disks. Detailed stellar population studies of nuclear stellar disks, combined with measurements of the star formation rates in the centers of dusty early-type galaxies, could test the consistency of this simple evolutionary model. A similar evolutionary scenario has been also proposed by Storchi-Bergmann et al. (2001) for Seyfert galaxies, in order to explain the incidence of recent star formation in the circumnuclear region of $40 \%$ of the galaxies of their sample.

\section{CONCLUSIONS}

We have used archival $H S T$ WFPC 2 images of 34 active and 34 inactive, early-type galaxies $(T<0)$ to investigate if activity is correlated with the presence of circumnuclear dust. We found a strong correlation between the presence of circumnuclear (hundreds of parsecs scale) dust and nuclear activity: all earlytype AGN hosts have circumnuclear dust, while only $26 \%$ (9 of 34) early-type, inactive hosts have circumnuclear dust. This indicates that the presence of circumnuclear dust is a requirement, although not a sufficient condition, of black hole accretion in early-type galaxies. The circumnuclear dust in all of these earlytype galaxies is typically confined to the central kiloparsec and is not observed on larger scales. The morphology of the circumnuclear dust is also typically complex, although a minority of galaxies $(<25 \%)$ show well-defined dusty disks with tightly wound spiral structure. Of the inactive galaxies, $38 \%$ (13) have structures that appear to be nuclear stellar disks. As these structures appear to share the same position angle as the host galaxy, these structures 
are more likely to be stellar disks than nuclear stellar bars. We also investigated a sample of 31 active and 31 inactive late-type galaxies $(T \geq 0)$ and found that all of the galaxies show evidence for circumnuclear dust, independent of the presence of nuclear accretion.

While our data demonstrate a clear connection between dust and AGNs in early-type galaxies, the origin of the dust and therefore the nature of the AGN-fueling mechanism remains unclear. We consider both internal and external origins for the circumnuclear dust, but both present significant challenges. Internal dust creation via stellar mass loss appears to be inconsistent with the absence of dust in approximately $50 \%$ of all early-type galaxies. An external origin, in contrast, appears to require frequent mergers of small gas and dust-rich galaxies. The timescale of these mergers is set by the settling and destruction times of the dust, which may be as short as a few dynamical times for these kiloparsec or smaller structures, or $10^{8} \mathrm{yr}$. The large observed fraction of early-type galaxies with nuclear stellar disks, the even larger fraction of early-type galaxies with dusty disks, and the relatively short lifetime of the dust appears to require continual growth and replenishment of nuclear stellar disks. Our observations demonstrate that this process is also intimately linked with some accretion onto the central, supermassive black hole. Observations of the dust kinematics and mass, along with improved models of the dust settling and destruction at the centers of early-type galaxies, could resolve the origin of the dust and nuclear stellar disks and reveal the fueling mechanism for these low-luminosity AGNs.

We would like to thank Barbara Ryden for helpful discussions. R. S. L., T. S. B, and M. F. S. acknowledge support from the Brazilian institutions CNPq, CAPES, and FAPERGS. Support for this work was also provided by NASA through grant AR-10677 from the Space Telescope Science Institute, which is operated by the Association of Universities for Research in Astronomy, Inc., under NASA contract NAS 5-26555.
Athey, A., Bregman, J., Temi, P., \& Sauvage, M. 2002, ApJ, 571, 272

Bender, R., Döbereiner, S., \& Möllenhoff, C. 1988, A\&AS, 74, 385

Bertola, F., Bettoni, D., Rusconi, L., \& Sedmak, G. 1984, AJ, 89, 356

Bertola, F., Buson, L. M., \& Zeilinger, W. W. 1992, ApJ, 401, L79

Capaccioli, M., Held, E. V., \& Nieto, J.-L. 1987, AJ, 94, 1519

Cappellari, M., et al. 2006, MNRAS, 366, 1126

Erwin, P., \& Sparke, L. S. 2002, AJ, 124, 65

Fathi, K., Storchi-Bergmann, T., Riffel, R. A., Winge, C., Axon, D. J., Robinson, A., Capetti, A., \& Marconi, A. 2006, ApJ, 641, L25

Ferrarese, L., \& Merrit, D. 2000, ApJ, 539, L9

Ferrarese, L., et al. 2006, ApJS, 164, 334

Gebhardt, K., et al. 2000, ApJ, 539, L13

Ho, L. C., Filippenko, A. V., \& Sargent, W. L. W. 1995, ApJS, 98, 477 1997a, ApJS, 112, 315 1997b, ApJ, 487, 568

Jaffe, W., Ford, H. C., O'Connell, R. W., van den Bosch, F., \& Ferrarese, L. 1994, AJ, 108, 1567

Knapp, G. R., Gunn, J. E., \& Wynn-Williams, C. G. 1992, ApJ, 399, 76

Kormendy, J., \& Djorgovski, S. 1989, ARA\&A, 27, 235

Kormendy, J., et al. 2005, AJ, 129, 2636

Krajnović, D., \& Jaffe, W. 2004, A\&A, 428, 877

Krist, J., \& Hook, R. 2004, The Tiny Tim User's Guide, Version 6.3 (Baltimore: STScI)

Lauer, T. R., et al. 2005, AJ, 129, 2138

Malkan, M. A., Gorjian, V., \& Tam, R. 1998, ApJS, 117, 25

Marston, A. P. 1988, MNRAS, 231, 333

Martini, P. 2004, in IAU Symp. 222, The Interplay among Black Holes, Stars and ISM in Galactic Nuclei, ed. Th. Storchi-Bergmann, L. C. Ho, \& H. R. Schmitt (Cambridge: Cambridge Univ. Press), 235

Martini, P., \& Pogge, R. W. 1999, AJ, 118, 2646

Martini, P., Regan, M. W., Mulchaey, J. S., \& Pogge, R. W. 2003, ApJ, 589, 774

Mathews, W. G., \& Brighenti, F. 2003, ApJ, 590, L5

Morelli, L., et al. 2004, MNRAS, 354, 753

\section{REFERENCES}

Morganti, R., et al. 2006, MNRAS, 371, 157

Peeples, M., \& Martini, P. 2006, ApJ, 652, 1097

Pizzella, A., Corsini, E. M., Morelli, L., Sarzi, M., Scarlata, C., Stiavelli, M., \& Bertola, F. 2002, ApJ, 573, 131

Pogge, R. W., \& Martini, P. 2002, ApJ, 569, 624

Prieto, M. A., Maciejewski, W., \& Reunanen, J. 2005, AJ, 130, 1472

Ravindranath, S., Ho, L. C., Peng, C. Y., Filippenko, A. V., \& Sargent, W. L. 2001, AJ, 122, 653

Regan, M. W., \& Mulchaey, J. S. 1999, AJ, 117, 2676

Rest, A., et al. 2001, AJ, 121, 2431

Rix, H.-W., \& White, S. D. M. 1990, ApJ, 362, 52

Ryden, B. S. 1992, ApJ, 396, 445

Sadler, E. M., \& Gerhard, O. E. 1985, MNRAS, 214, 177

Sarzi, M., et al. 2006, MNRAS, 366, 1151

Seifert, W., \& Scorza, C. 1996, A\&A, 310, 75

Scorza, C., \& Bender, R. 1995, A\&A, 293, 20

Scorza, C., \& van den Bosch 1998, MNRAS, 300, 469

Storchi-Bergmann, T., Gonzalez Delgado, R., Schmitt, H. R., Cid Fernandes, R., \& Heckman, T. M. 2001, ApJ, 559, 147

Storchi-Bergmann, T., Nemmen, R. S., Spinelli, P. F., Eracleous, M., Wilson, A. S., Filippenko, A. V., \& Livio, M. 2005, ApJ, 624, L13

Temi, P., Brighenti, F., Mathews, W. G., \& Bregman, J. D. 2004, ApJS, 151, 237

Tran, H. D., Tsvetanov, Z., Ford, H. C., Davies, J., Jaffe, W., van den Bosch, F. C., \& Rest, A. 2001, AJ, 121, 2928

Tremaine, S., et al. 2002, ApJ, 574, 740

van den Bosch, F. C., Jaffe, W., \& van der Marel, R. P. 1998, MNRAS, 293, 343

van Dokkum, P. G., \& Franx, M. 1995, AJ, 110, 2027

Verdoes Kleijn, G. A., Baum, S. A., de Zeeuw, P. T., \& O’Dea, C. P. 1999, AJ, 118,2592

Verdoes Kleijn, G. A., \& de Zeeuw, P. T. 2005, A\&A, 435, 43

Vincent, R. A., \& Ryden, B. S. 2005, ApJ, 623, 137

Xilouris, E. M., \& Papadakis, I. E. 2002, A\&A, 387, 441 